\title{
ON SERRE DUALITY AND ENVELOPES OF HOLOMORPHY
}

\author{
BY \\ HENRY B. LAUFER $\left({ }^{1}\right)$
}

1. Introduction. Serre [15] established that, under appropriate conditions, $H_{*}^{n-q}\left(D, \Omega^{n-p}\right) \approx\left[H^{q}\left(D, \Omega^{p}\right)\right]^{*}$, where $D$ is an $n$-dimensional, $\sigma$-compact complex manifold, $\Omega^{r}$ is the sheaf of germs of holomorphic $r$-forms, and $H_{*}^{p}$ stands for cohomology with compact supports. As Serre shows, when $D$ is not compact, the above duality need not hold. In this paper, we derive a formula which is valid in all cases.

Specifically, we put natural topologies on all the cohomology groups, making the groups linear topological spaces. Then (Theorem 3.1)

$$
H_{*}^{n-q}\left(D, \Omega^{n-p}\right) \approx\left[H^{q}\left(D, \Omega^{p}\right)\right]^{*} \oplus R^{q+1}\left(D, \Omega^{p}\right)
$$

and

$$
H^{n-q}\left(D, \Omega^{n-p}\right) \approx\left[H^{q}\left(D, \Omega^{p}\right)\right]^{*} \oplus R^{q+1}\left(D, \Omega^{p}\right),
$$

where $R^{q+1}$ and $R_{*}^{q+1}$ have indiscrete (trivial) topologies. $R^{q+1}$ measures the extent to which $\bar{\partial}_{q+1}$ is a relatively open map. We show that $R^{q+1}\left(D, \Omega^{p}\right)=0$ if and only if $H^{q+1}\left(D, \Omega^{p}\right)$ has a Hausdorff topology. Similarly, $R_{*}^{q+1}\left(D, \Omega^{p}\right)=0$ if and only if $H_{*}^{q+1}\left(D, \Omega^{p}\right)$ has a Hausdorff topology. There is an analogous result (Theorem 3.2) for complex vector bundles.

We then let $D$ be an open subset of the Stein manifold $M$. Let $X=M-D$. The principal result (Theorem 4.7) is that $M$ is the envelope of holomorphy of $D$ if and only if $H_{*}^{n}\left(X, \Omega^{n}\right)=0$ and $H_{*}^{n-1}\left(X, \Omega^{n}\right)$ has an indiscrete topology. Here we can use either a natural topology on $H_{*}^{n-1}\left(X, \Omega^{n}\right)$ or its topology as a subset of $H_{*}^{n}\left(D, \Omega^{n}\right)$ (Theorem 4.12). We also show that $H^{n-1}\left(D, \Omega^{p}\right)$ is either 0 - or infinitedimensional (Theorem 4.5) and give a new proof of the Bochner-Martinelli integral formula.

Much of the research for this article was done at Princeton University and appeared in the author's doctoral dissertation. The author would like to thank Robert C. Gunning for his supervision and help during that period.

We shall use the terminology of [7] for linear topological spaces and assume [5] as background for the complex analysis.

Received by the editors October 12, 1966.

(1) Part of this research was done while the author was a National Science Foundation Graduate Fellow. It was also partially sponsored by the Air Force Office of Scientific Research, Office of Aerospace Research, United States Air Force, under AFOSR Grant No. 335-63. 
2. Natural topologies for the cohomology groups. We consider three different ways to calculate cohomology, by Leray coverings, by $C^{\infty}$ differential forms, and by currents (differential forms with distributions as coefficients, see [14] and [12]). Sheaf theoretical considerations show that all yield the same cohomology groups, as vector spaces. In this section we show that the various induced topologies are identical.

We recall that the strong topology for the dual $S^{*}$ of the linear topological space $S$ is the topology of uniform convergence on bounded sets $T \subset S$. That is, a base for the neighborhoods of the origin may be given by sets of the form $\left\{f \in S^{*}|| f(t) \mid<\varepsilon, t \in T\right.$ and $\left.\varepsilon>0\right\} ; T$ is bounded if given any neighborhood $U$ of the origin in $S$, there is a nonzero scalar $a$ such that $a T \subset U$. We also note that to calculate cohomology with compact supports on a $\sigma$-compact manifold, we can take a locally finite Leray covering by relatively compact simplices. Then $q$-cochains with compact support correspond to elements of the direct sum of the spaces of sections of the various $q$-simplices. The usual diagram chase in the spectral diagram, taking care to keep compact support, shows that the cohomology derived from the Leray covering is algebraically identical to the cohomology with compact supports computed from, say, a resolution by $C^{\infty}$ differential forms.

We shall say that $H^{q}\left(D, \Omega^{p}\right)$ has the strong topology with respect to a Leray covering $\mathfrak{U}$ if we give the $q$-cochains of $N(\mathfrak{U})$ the topology of uniform convergence on compact sets on each simplex and then take the direct product with the direct product topology. We recall that if $S=\chi S_{i}$ expresses $S$ as a direct product, a base for the neighborhoods of the origin in the product topology may be given by sets of the form $\left\{s \in S \mid p_{i}(s) \in U_{i}, 1 \leqq i \leqq n\right\}$ where $p_{i}: S \rightarrow S_{i}$ is the projection map and $U_{i}$ is a neighborhood of the origin in $S_{i}$. Then we give the $q$-cocycles the subspace topology and $H^{q}\left(D, \Omega^{p}\right)$ the quotient topology.

For $H_{*}^{q}\left(D, \Omega^{p}\right)$ take the direct sum topology on the direct sum. If $S=\sum S_{t}$ expresses $S$ as a direct sum, a base for the neighborhoods of the origin in the direct sum topology is given by those convex sets $V$ such that $i_{t}^{-1}(V)$ is a neighborhood of the origin in $S_{t}$ for all $t$. Here $i_{t}: S_{t} \rightarrow S$ is the injection. Equivalently, the direct sum topology is the strongest locally convex topology such that the injections $\left\{i_{t}\right\}$ are all continuous. The box topology on the direct sum has a local base for neighborhoods of the origin consisting of sets of the form $V=\left\{s \in S \mid p_{t}(s) \in U_{t}\right.$ for all $\left.t\right\}$ where $p_{t}: S \rightarrow S_{t}$ is the projection map and $U_{t}$ is a neighborhood of the origin in $S_{t}$. We shall show in Lemma 2.1 below that the box and direct sum topologies coincide for countable direct sums.

We recall that if $T$ is a locally convex topology for $S$, the weak topology on $S$ (with respect to $T$ ) is the topology generated by the continuous linear functionals on $S$ with the topology $T$. A base for neighborhoods of the origin for the weak topology is given by sets of the form $\left\{s \in S|| f_{j}(s) \mid<\varepsilon, 1 \leqq j \leqq n, f_{j} \in S^{*}\right\}$. We shall say that $H^{q}\left(D, \Omega^{p}\right)$ has the weak topology with respect to a Leray covering if we give the direct product its weak topology with respect to the direct product 
topology. The weak topology on $H^{q}\left(D, \Omega^{p}\right)$ as thus computed is identical with the weak topology on $H^{q}\left(D, \Omega^{p}\right)$ with respect to the strong topology on $H^{q}\left(D, \Omega^{p}\right)$ [7, Theorem 17.13, p. 160]. Similarly, we have a weak topology for $H_{*}^{q}\left(D, \Omega^{p}\right)$.

$C^{\infty}$ forms and currents have strong topologies given by, for the former, uniform convergence on compact sets for all derivatives of the coefficients when we write the forms in terms of a coordinate covering (the $C^{\infty}$ topology), and for the latter, the strong topology as a dual space. We may also give $C^{\infty}$ forms their weak topology with respect to the $C^{\infty}$ topology and may give currents their weak topology. These yield strong and weak topologies for $H^{q}\left(D, \Omega^{p}\right)$.

Similarly, we can give $C^{\infty}$ forms with compact support the Schwartz topology or the weak topology with respect to the Schwartz topology. Also, currents with compact support have both strong and weak topologies. These all yield strong and weak topologies for $H_{*}^{q}\left(D, \Omega^{p}\right)$. The Schwartz topology may be described as follows. Write $D=\bigcup_{n=1}^{\infty} K_{n}$ with $K_{n} \subset K_{n+1}$ and $K_{n}$ compact. Consider all the $C^{\infty}$ forms ${ }_{n} E_{*}$ with support in $K_{n}$. Give ${ }_{n} E_{*}$ the $C^{\infty}$ topology, i.e., the topology which it receives as a subset of the $C^{\infty}$ forms when these have the $C^{\infty}$ topology. $E_{*}$, the set of $C^{\infty}$ forms with compact support, is the union of the ${ }_{n} E_{*}$. The Schwartz topology on $E_{*}$ is the inductive limit topology, i.e., a basis for the neighborhoods of the origin is given by convex sets $V$ such that $V \cap{ }_{n} E_{*}$ is open in ${ }_{n} E_{*}$. An equivalent topology is given by the following nondenumerable set (2.1) of seminorms. (See [14, Theorem II, pp. 65-67] for a proof when $D=\boldsymbol{R}^{n}$. Schwartz's proof may be modified to apply to any manifold $D$.) Let $\left\{\Omega_{0}=\varnothing, \Omega_{1}, \Omega_{2}, \ldots, \Omega_{v}, \ldots\right\}$ be an infinite sequence of relatively compact open sets in $D$ such that $D=\cup \Omega_{v}$ and $\bar{\Omega}_{v} \subset \Omega_{v+1}$. Let $\{\varepsilon\}=\left\{\varepsilon_{0}, \varepsilon_{1}, \ldots\right\}$ be a decreasing sequence of positive numbers which approach 0 . Let $\{m\}=\left\{m_{0}, m_{1}, \ldots, m_{v}, \ldots\right\}$ be an increasing sequence of nonnegative integers which approach $\infty$. For each pair $\{m\},\{\varepsilon\}$, we get a seminorm

$$
\left.N(\{m\} ;\{\varepsilon\})(\phi)=\sup _{v} \sup _{x \notin \Omega_{\nu} ;|p| \leqq m}\left|D^{p} \phi(x)\right| / \varepsilon\right)
$$

where $\phi$ is a $C^{\infty}$ function, $p$ a multi-index and $D^{p}$ must be taken with respect to a fixed coordinate covering. For a $C^{\infty}$ form, let $\{\phi\}$ be the coefficients and apply $N(\{m\} ;\{\varepsilon\})$ to each one and take the supremum.

$\alpha: A \rightarrow B$, a linear mapping between the locally convex linear topological spaces $A$ and $B$, is a homomorphism if it is continuous and relatively open. $\alpha$ is then necessarily a homomorphism in the weak topologies for $A$ and $B$ since the weak topology on $A / \operatorname{ker} \alpha$ is the quotient of the weak topology on $A$ [7, Theorem 17.13 (ii), p. 160] and is also identical with its weak topology when thought of as a subspace of $B$ [7, Theorem 17.13 (i), p. 160].

We shall say that a sequence $A \stackrel{\alpha}{\longrightarrow} B \stackrel{\beta}{\longrightarrow} C$ is topologically exact if it is algebraically exact and if $\alpha$ and $\beta$ are homomorphisms.

THEOREM 2.1. The strong topologies for $H^{q}\left(D, \Omega^{p}\right)$ and $H_{*}^{q}\left(D, \Omega^{p}\right)$ with respect 
to a Leray covering, $C^{\infty}$ forms, and currents all coincide. Similarly, the weak topologies for $H^{q}\left(D, \Omega^{p}\right)$ and $H_{*}^{q}\left(D, \Omega^{p}\right)$ all coincide.

Proof. Form the usual diagram, such as diagram (2.2). For currents, instead of $E^{p, q}$, use $K^{p, q}$, where $K^{p, q}$ is the sheaf of germs of currents of type $(p, q)$.

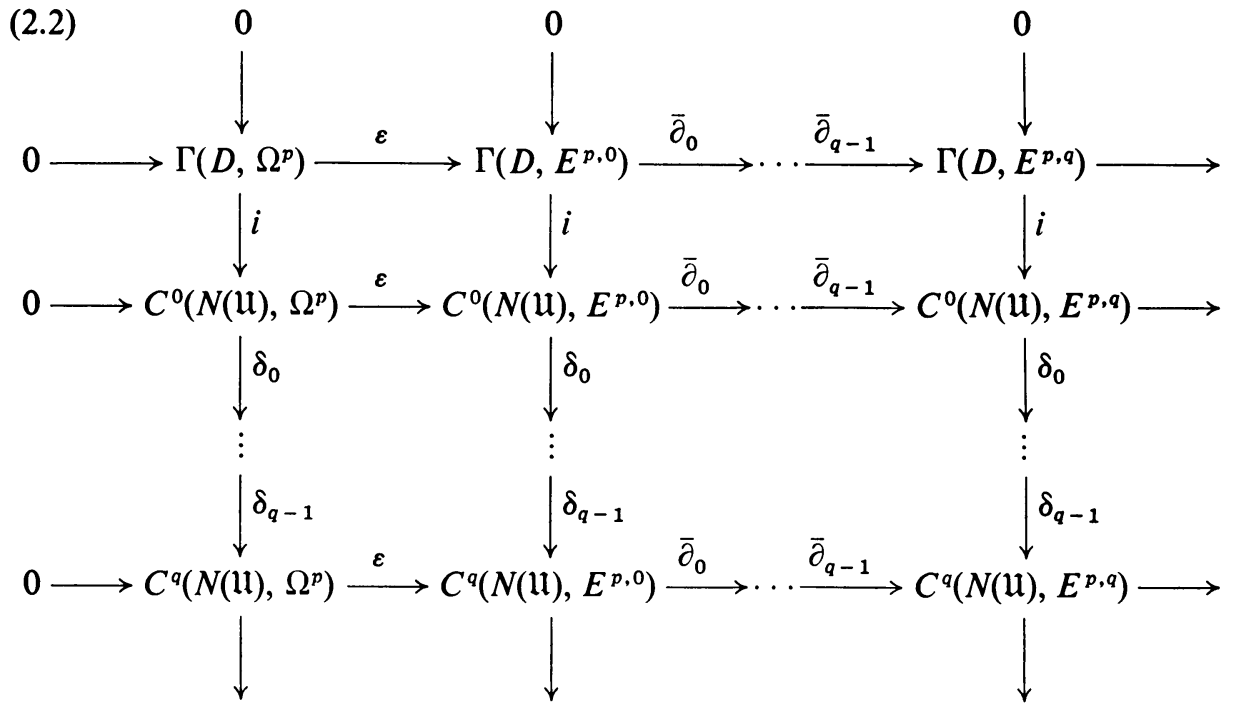

Let $H^{q}\left(N(\mathfrak{l}), \Omega^{p}\right)$ stand for cohomology as computed from the Leray covering and $H^{q}\left(D, \Omega^{p}\right)$ stand for cohomology as computed from $C^{\infty}$ differential forms (or from currents). (2.2) is the usual diagram for deriving the algebraic isomorphism $H^{q}\left(D, \Omega^{p}\right) \approx H^{q}\left(N(\mathfrak{l}), \Omega^{p}\right)$.

$$
\theta: H^{q}\left(N(\mathfrak{l}), \Omega^{p}\right) \rightarrow H^{q}\left(D, \Omega^{p}\right)
$$

and

$$
\theta^{\prime}: H^{q}\left(D, \Omega^{p}\right) \rightarrow H^{q}\left(H(11), \Omega^{p}\right)
$$

are isomorphisms given by

$$
\theta=\pi_{q} \circ i^{-1} \circ \bar{\partial}_{q-1} \circ \delta_{0}^{-1} \circ \ldots \circ \bar{\partial}_{0} \circ \delta_{q-1}^{-1} \circ \varepsilon \circ\left(\pi_{q}^{\prime}\right)^{-1}
$$

and

$$
\theta^{\prime}=\pi_{q}^{\prime} \circ \varepsilon^{-1} \circ \delta_{q-1} \circ \bar{\partial}_{0}^{-1} \circ \ldots \circ \delta_{0} \circ \bar{\partial}_{q-1}^{-1} \circ i \circ \pi_{q}^{-1} .
$$

Here $\pi_{q}: \operatorname{ker} \bar{\partial}_{q} \rightarrow H^{q}\left(D, \Omega^{p}\right)$ and $\pi_{q}^{\prime}: \operatorname{ker} \delta_{q} \rightarrow H^{q}\left(N(\mathfrak{l}), \Omega^{p}\right)$ are the projection maps. It suffices to show that $\theta$ and $\theta^{\prime}$ are continuous.

We claim that it is sufficient in all cases (i.e., with and without compact support and in the strong and the weak topologies) to show that the maps $i, \varepsilon$ and those $\delta$ and $\bar{\partial}$ which are not in the first row or column are all homomorphisms. For example, consider $\theta$. $\theta$ is induced by the map on the cocycles, $Z^{q}\left(N(\mathfrak{l}), \Omega^{p}\right)$. The 
injection $\varepsilon$ is continuous. Since $\delta_{q-1}$ is assumed relatively open, there is a continuous (in fact an isomorphic) map

$$
\delta_{q-1}^{-1}: \operatorname{im} \delta_{q-1} \rightarrow C^{q-1}\left(N(\mathfrak{U}), E^{p, 0}\right) / \operatorname{ker} \delta_{q-1} .
$$

Since $\varepsilon\left(Z^{q}\left(N(\mathfrak{u}), \Omega^{p}\right)\right) \subset \mathrm{im} \delta_{q-1}$ by exactness,

$$
\delta_{q-1}^{-1} \circ \varepsilon: Z^{q}\left(N(\mathfrak{U}), \Omega^{p}\right) \rightarrow C^{q-1}\left(N(\mathfrak{u}), E^{p, 0}\right) / \operatorname{ker} \delta_{q-1} .
$$

$\bar{\partial}_{0}$ is continuous and so induces a continuous map

$$
\bar{\partial}_{0}^{\prime}: C^{q-1}\left(N(\mathfrak{u}), E^{p, 0}\right) \rightarrow C^{q-1}\left(N(\mathfrak{u}), E^{p, 1}\right) / \operatorname{im} \bar{\partial}_{0} \circ \delta_{q-2} .
$$

The kernel of $\bar{\partial}_{0}^{\prime}$ contains the image of $\delta_{q-2}$, which by exactness is the kernel of $\delta_{q-1}$. Hence $\bar{\partial}_{0}^{\prime}$ induces a continuous map

$$
C^{q-1}\left(N(\mathfrak{u}), E^{p, 0}\right) / \operatorname{ker} \delta_{q-1} \rightarrow C^{q-1}\left(N(\mathfrak{u}), E^{p, 1}\right) / \operatorname{im} \bar{\partial}_{0} \circ \delta_{q-2} .
$$

Hence $\bar{\partial}_{0} \circ \delta_{q-1}^{-1} \circ \varepsilon$ is continuous. At $C^{q-1}\left(N(\mathfrak{u}), E^{p, 1}\right)$ we have, as above, a continuous map

$$
\bar{\partial}_{1} \circ \delta_{q-2}^{-1}: \operatorname{im} \delta_{q-2} \rightarrow C^{q-2}\left(N(\mathfrak{u}), E^{p, 2}\right) / \operatorname{im} \bar{\partial}_{1} \circ \delta_{q-3} .
$$

$\operatorname{im} \bar{\partial}_{0} \circ \delta_{q-2}$ is in the kernel of $\bar{\partial}_{1} \circ \delta_{q-2}^{-1}$ so that $\bar{\partial}_{1} \circ \delta_{q-2}^{-1} \circ \bar{\partial}_{0} \circ \delta_{q-1}^{-1} \circ \varepsilon$ is well defined and continuous. We may continue in this fashion until we reach $\bar{\partial}_{q-1} \circ \delta_{0}^{-1}$. Our target space is $C^{0}\left(N(\mathfrak{U}), E^{p, q}\right) / \operatorname{im} \bar{\partial}_{q-1} \circ i$ and we know that the image lies in $\operatorname{ker} \delta_{0} / \mathrm{im} \bar{\partial}_{q-2} \circ i$. This last image is just $H^{q}\left(D, \Omega^{p}\right)$ and the topologies coincide because $i$ is a homomorphism.

Finally, as mentioned above, to show that $\varepsilon, i, \bar{\partial}$, and $\delta$ are homomorphisms in the weak topologies it suffices to show them to be homomorphisms in the strong topologies. This is the purpose of the next five lemmas. If one initially puts the box topology on the direct sum, Lemma 2.1 becomes unnecessary.

LeMmA 2.1. Let $S=\sum_{j=1} S_{j}$ be a countable direct sum of linear topological spaces $S_{j}$. Then the direct sum and box topologies coincide for $S$.

Proof. Let $U$ be a box neighborhood of the origin in $S$. We may assume that $U=\left\{s \in S \mid s_{j} \in U_{j}, 1 \leqq j<\infty\right\}$ where $U_{j}$ is a neighborhood of the origin in $S_{j}$ and $s_{j}=p_{j}(s)$ is the image of $s$ under the projection map $p_{j}: S \rightarrow S_{j}$. $U$ is convex and letting $i_{j}: S_{j} \rightarrow S$ be the injection, $i_{j}^{-1}(U)=U_{j}$ is open in $S_{j}$. Hence $U$ is a neighborhood of the origin in the direct sum topology.

Conversely, let $U$ be a neighborhood of the origin in the direct sum topology. Then we may assume that $U$ is convex and that $i_{j}^{-1}(U)=U_{j}$ is a neighborhood of the origin in $S_{j}$.

Let $U_{j}^{\prime}=\left(1 / 2^{j}\right) U_{j}, 1 \leqq j<\infty$. We claim that $U^{\prime}=\left\{s \in S \mid s_{j} \in U_{j}^{\prime}, 1 \leqq j<\infty\right\} \subset U$. Verification will show that $U$ is a neighborhood of the origin in the box topology 
and will complete the proof of the lemma. Let $s \in U^{\prime}$. Then $s=s_{j_{1}} \oplus \cdots \oplus s_{j_{n}}$ with $s_{j} \in U_{j}^{\prime}$.

$$
s=\frac{1}{2^{j_{1}}}\left(2^{j_{1}} s_{j_{1}}\right) \oplus \cdots \oplus \frac{1}{2^{j_{n}}}\left(2^{j_{n}} s_{j_{n}}\right)
$$

and

$$
\begin{aligned}
t & =\sum_{i} \frac{1}{2^{j_{t}}}<1 \\
s=t \cdot \frac{s}{t} & =t\left\{\frac{1}{t 2^{j_{1}}}\left(2^{j^{j}}{ }_{j_{j_{1}}}\right) \oplus \cdots \oplus \frac{1}{t 2^{j_{n}}}\left(2^{j_{n}} s_{j_{n}}\right)\right\} .
\end{aligned}
$$

Looking inside the braces, we see that $s / t$ is a convex combination of the $i_{j_{v}}\left(2^{j_{v}} s_{j_{v}}\right) \in i_{j_{v}}\left(U_{j_{v}}\right) \subset U$. Since $U$ is convex, $s / t \in U$. Since $U$ contains the origin and is convex and since $t<1, s \in U$.

LEMMA 2.2. $\varepsilon$ is a homomorphism in the strong topologies for the direct sum and the direct product.

Proof. It suffices to prove that $\varepsilon$ is a homomorphism when we restrict to a single simplex since we may use the box topology on the direct sum.

For the case of $C^{\infty}$ forms, uniform convergence on compact sets of holomorphic functions implies uniform convergence on compact sets of all derivatives. Hence $\varepsilon$ is a homomorphism.

For currents it suffices to restrict ourselves to coordinate neighborhoods. Let $S=U_{0} \cap \cdots \cap U_{q}$ be a $q$-simplex. An element $\phi \in \Gamma\left(S, \Omega^{p}\right)$ is given by

$$
\phi=\sum f_{i_{1}} \cdots i_{p} d z_{i_{1}} \wedge \cdots \wedge d z_{i_{p}}
$$

where $f_{i_{1}} \ldots i_{p}$ is holomorphic. Test forms, of type $(n, n-p)$, must be $C^{\infty}$ and with compact support. A bounded set $T$ must have common support for the coefficients and a common bound on each derivative [14, Theorem IV, p. 69]. The injection of $\phi$ into the set of currents is given by taking $\langle\phi, g\rangle=\int_{S} \phi \wedge g$. To see that $\varepsilon$ is continuous, consider a sequence $\phi_{i}$ of elements of $\Gamma\left(S, \Omega^{p}\right)$ which approach 0 . Given any bounded set $T$ of test functions, $\left|g_{i}\right|<M$ for $g_{i}$ a coefficient of an element $g \in T$. The $g_{i}$ have common support $K$. Hence, since the coefficients of the $\phi_{i}$ approach 0 uniformly on $K,\left\langle\phi_{i}, g\right\rangle$ approaches 0 uniformly.

To prove $\varepsilon$ relatively open, we may work separately on each coefficient. For simplicity, we assume that $\Omega^{p}=\mathcal{O}$, the sheaf of germs of holomorphic functions. In writing test forms with compact support, we shall just write the coefficient. It suffices to show that if a net $\left\{f_{\alpha}\right\}_{\alpha \in A} \rightarrow 0$ as a net of distributions, then $\left\{f_{\alpha}\right\} \rightarrow 0$, as holomorphic functions.

Let $\bar{\Delta}=\{0, \rho\}$ be a closed polydisc with $2 \Delta$ contained in our given coordinate neighborhood. The Cauchy integral formula gives

$$
f_{\alpha}(z)=\left(\frac{1}{2 \pi i}\right)^{n} \int_{\left|u_{j}-\zeta_{j}\right|=\rho} \frac{f(\zeta) d \zeta_{1} \cdots d \zeta_{n}}{\left(\zeta_{1}-z_{1}\right) \cdots\left(\zeta_{n}-z_{n}\right)} .
$$


Let $\zeta=\left(\zeta_{1}, \ldots, \zeta_{n}\right)=\left(r_{1} e^{i \theta_{1}}, \ldots, r_{n} e^{i \theta_{n}}\right)=r e^{i \theta}$. From above,

$$
f_{\alpha}(z)=\left(\frac{1}{2 \pi}\right)^{n} \int_{0 \leqq \theta_{i} \leqq 2 \pi} f\left(z+r e^{i \theta}\right) d \theta_{i} \cdots d \theta_{n} .
$$

We want a test function roughly like $t(z)=r_{1} \cdots r_{n}$. Let $t_{v}(z)=\tau\left(r_{v}\right)$ with $\tau\left(r_{v}\right) C^{\infty}$ and with nontrivial support in the interval $\rho / 2 \leqq r_{v} \leqq 3 \rho / 2$. Let $t(z)=\prod_{v=1}^{n} t_{v}(z)$. Then $t(z)$ is independent of $\theta$.

$$
\begin{aligned}
f_{\alpha}(z) \int_{0 \leqq r_{i} \leqq 2 \rho} t d r_{1} \cdots d r_{n}= & \int_{0 \leqq r_{i} \leqq 2 \rho} f_{\alpha}(z) t d r_{1} \cdots d r_{n} \\
= & \left(\frac{1}{2 \pi}\right)^{n} \int_{0 \leqq r_{i} \leqq 2 \rho} \int_{0 \leqq \theta_{i} \leqq 2 \pi} f_{\alpha}\left(z+r e^{i \theta}\right) t / \Pi r_{i} \\
& \times d \theta_{1} \cdots d \theta_{n} r_{1} d r_{1} \cdots r_{n} d r_{n} \\
= & \left(\frac{1}{2 \pi}\right)^{n}\left\langle f_{\alpha}, t / \Pi r_{i}\right\rangle .
\end{aligned}
$$

From (2.3) we obtain

$$
f_{\alpha}(z)=\left(1 / \int t d r_{1} \cdots d r_{n}\right) \frac{1}{(2 \pi)^{n}}\left\langle f_{\alpha}, t / \Pi r_{i}\right\rangle
$$

Since the net $\left\{f_{\alpha}\right\}$ converges, the right side of (2.4) converges. But in fact we may translate our test function (and $r$ ) a little and still retain a bounded set of test functions since we can retain common compact support. The right side of (2.4) converges for bounded sets of test functions and thus the left side of (2.4) converges uniformly. Since $S$ is $\sigma$-compact, this implies uniform convergence on compact sets.

Lemma 2.3. $i$ is a homomorphism in the strong topologies for the direct sum and the direct product.

Proof. First for $C^{\infty}$ forms. An open set in $C^{0}\left(N(\mathfrak{U}), E^{p, t}\right)$ consists of chains with norm conditions on a finite number of simplices so that $i$ is continuous. To see that $i$ is relatively open, take for instance $W=\left\{f \in \Gamma\left(D, E^{p . t}\right) \mid\|f\|_{K}<\varepsilon\right\}$. Then $K$ can be covered by a finite number of open sets $W_{i}$ such that $W_{i}$ is a relatively compact subset of some element $U_{i} \in \mathfrak{l}$. Let $V \in C^{0}\left(N(\mathfrak{l}), E^{p . t}\right)$ be

$$
\left\{g \in C^{0}\left(N(\mathfrak{U}), E^{p, t}\right) \mid\|g\|_{\bar{w}_{\mathfrak{t}}}<\varepsilon\right\} .
$$

$V$ is an open set such that $i(W) \supset\left(V \cap i\left(\Gamma\left(D, E^{p, t}\right)\right)\right.$ so that $i$ is relatively open.

For $C^{\infty}$ forms with compact support we think of the Schwartz topology as the inductive limit topology taken over $C^{\infty}$ forms with fixed compact support. Then to prove $i$ continuous we need only prove continuity on each set of $C^{\infty}$ forms with common support. Since a finite number of simplices in $N(\mathfrak{l l})$ are involved, $i$ is continuous.

To get an open set $W$ in $\Gamma_{*}\left(D, E^{p . t}\right)$ use the seminorms (2.1) to define $W=\left\{f \in \Gamma_{*}\left(D, E^{p . t}\right) \mid N(\{m\} ;\{\varepsilon\})(f)<1\right\}$. The condition $N(\{m\} ;\{\varepsilon\})(f)<1$ puts 
only a finite number of conditions on $f$ restricted to the compact sets $\bar{\Omega}_{v}-\Omega_{v-1}$. As above, cover the $\bar{\Omega}_{v}-\Omega_{\nu-1}$ by locally finite sets $W_{i}$ such that the $W_{i}$ are open and each is a relatively compact subset of some element $U_{i} \in \mathfrak{l l}$. Then each simplex $U_{i}$ contains only a finite number of the $W_{i}$ and thus $i$ is a relatively open map.

For currents with arbitrary support, in the direct product we have bounded sets $T_{i}$ of test functions for a finite number of simplices. $\cup T_{i}$ is a bounded set of test functions for $\Gamma\left(D, K^{p, t}\right)$ since they have support within a fixed compact set and common bounds on all derivatives. Hence $i$ is continuous. To prove $i$ relatively open, take a bounded set $S$ of test functions, with support within the compact set $K$. Take a $C^{\infty}$ partition of unity $\left\{\rho_{\alpha}\right\}$ subordinate to $U$. Use test functions $\left\{\rho_{\alpha} S\right\}$. Since $K$ can meet only a finite number of simplices, we get an open set in $C^{0}\left(N(\mathfrak{u}), K^{p, t}\right)$.

For currents with compact supports, to show $i$ continuous consider an open set $V$ in $C_{*}^{0}\left(N(\mathfrak{u}), K^{p, t}\right)$. For each simplex $U_{i}, V$ has a bounded set $T_{i}$ of $C^{\infty}$ test functions with compact support. $S=\bigcup T_{i}$ provides a bounded set of test functions for $\Gamma_{*}\left(D, K^{p, t}\right)$ so that $i$ is continuous.

Finally, to show $i$ relatively open, let $S$ be a bounded set of test functions for $\Gamma_{*}\left(D, K^{p, t}\right) .\left\{\rho_{\alpha} S\right\}$ provides a bounded set of test functions for each simplex $U_{i}$ when we assign to $U_{i}$ those functions $\rho_{\alpha}$ with support in $U_{i}$.

LEMma 2.4. $\delta_{t}$ is a homomorphism for the direct product and the direct sum.

Proof. Let $f$ be a $t$-cochain.

$$
\delta_{t} f\left(U_{0} \cap \cdots \cap U_{t+1}\right)=\sum_{i=0}^{t+1}(-1)^{i} f\left(U_{0} \cap \cdots \cap \hat{U}_{i} \cap \cdots \cap U_{t+1}\right)
$$

so that $\delta_{t}$ is continuous for $C^{\infty}$ forms and currents in the direct product and the direct sum.

We examine the inverse map $\delta_{t-1}^{-1}$ mapping $t$ cocycles to $t-1$ cochains as given in [5, Lemma VI.D.3, p. 188]. Let $\mathfrak{B}=\left\{V_{i}\right\}$ be a locally finite refinement of $\mathfrak{u}$. For each $V_{i}, \rho\left(V_{i}\right) \in \mathfrak{U l}$ such that $\bar{V}_{i} \subset \rho\left(V_{i}\right)$. Let $n_{V_{i}}$ be a $C^{\infty}$ partition of unity subordinate to $\mathfrak{B}$. A $t$-cocycle $f \in C^{t}\left(N(\mathfrak{U}), E^{p . s}\right)$ or $f \in C^{t}\left(N(\mathfrak{U}), K^{p, s}\right)$ satisfies $\delta_{t} f=0 . f=\sum_{V_{i}}\left(n_{V_{i}} f\right)$. Introduce the $(t-1)$-cochain $g_{V_{i}} \in C^{p-1}\left(N(\mathfrak{l l}), E^{p, s}\right)$ or $g_{V_{\mathfrak{t}}} \in C^{p-1}\left(N(\mathfrak{U}), K^{p, s}\right)$ defined on any $(t-1)$-simplex $\sigma=U_{0} \cap \cdots \cap U_{t-1} \in N(\mathfrak{l} \mathfrak{l})$ by

$$
\begin{aligned}
g_{V_{t}} & =0 & & \text { if }|\sigma| \cap V_{i}=\varnothing, \\
& =(-1)^{p}\left(n_{V_{t}} f\right)\left(U_{0} \cap \cdots \cap U_{t-1} \cap \rho\left(V_{i}\right)\right) & & \text { if }|\sigma| \cap V_{i} \neq \varnothing .
\end{aligned}
$$

The cochain $g=\sum_{i} g_{V_{l}} \in C^{t-1}\left(N(\mathfrak{u}), E^{p . s}\right)$ or $g \in C^{t-1}\left(N(\mathfrak{l l}), K^{p, s}\right)$ is well defined and satisfies $\delta_{t-1} g=f$.

We note that $\delta_{t}^{-1}$ keeps compact support. To examine continuity, we first note that the refinement $\mathfrak{B}$ and the partition of unity $\left\{n_{V_{i}}\right\}$ can be chosen independently of the cochain. For $C^{\infty}$ forms, a compact set $K$ in a $(t-1)$-simplex meets only a 
finite number of $t$ simplices so that the choice of $g_{V_{i}}$ is continuous in both the direct product and the direct sum. Similarly for currents.

LEMMA 2.5. $\bar{\partial}$ is a homomorphism in the direct sum and direct product for $C^{\infty}$ forms. For currents, $\bar{\partial}$ is a homomorphism in the direct sum and direct product if each simplex is a domain in $\boldsymbol{C}^{n}$.

Proof. It suffices to prove the lemma on each simplex. For $C^{\infty}$ forms we have the open mapping theorem for Fréchet spaces, so that $\bar{\partial}$ is a homomorphism. For currents we must go back and examine the inverse map as given in [2, Lemma 1.2, p. 96]. We first want to verify that after suitable modifications and restriction mappings, this map is continuous.

We are in $\boldsymbol{C}^{m}$. Dolbeault considers a fundamental solution for the Laplacian. Let $s_{n}$ be the area of the unit $(n-1)$-sphere. Let $r(x, y)$ be Euclidean distance in real $2 m$-space. For $m>1$, let $g_{0}(x, y)=(2 m-2)^{-1} s_{2 m}^{-1} r^{2-2 m}(x, y)$. For $m=1$, let $g_{0}(x, y)=-(1 / 2 \pi) \log r(x, y)$. Let $\psi$ be a $C^{\infty}$ function with compact support.

$$
G_{0} \psi=\int g_{0}(x, y) \psi(y) d y
$$

is $C^{\infty}$ and $\Delta G_{0} \psi=\psi$. (2.5) may be extended to differential forms by operating on each coefficient. If $\theta$ is a current with compact support, let $\langle G \theta, \phi\rangle=\left\langle\theta, G_{0} \phi\right\rangle$, where $\phi$ is a $C^{\infty}$ differential form with compact support.

$$
\theta=2 d^{\prime \prime} \partial^{\prime \prime} G \theta+2 \partial^{\prime \prime} G d^{\prime \prime} \theta .
$$

In (2.6) we are using Dolbeault's notation, $d^{\prime \prime}=\bar{\partial}$ and $\partial^{\prime \prime}$ is a codifferentiation operator.

Now suppose we have a $\bar{\partial}$-closed current $t$ defined in the polyrectangle $S$. Multiply $t$ by a $C^{\infty}$ function $\eta$ which has compact support in $S$ but is identically 1 on, for instance, $(1-\varepsilon) S$. This is a continuous map from currents on $S$ to currents with compact support on $S$. Let $\theta=\eta t$. $d^{\prime \prime} \theta=0$ on the interior of $(1-\varepsilon) S=W^{\prime}$. $\Delta G d^{\prime \prime} \theta=d^{\prime \prime} \theta=0$ so that $G d^{\prime \prime} \theta$ is harmonic and hence (real) analytic on $W^{\prime}$. $d^{\prime \prime} \partial^{\prime \prime} G d^{\prime \prime} \theta=d^{\prime \prime} \partial^{\prime \prime} d^{\prime \prime} G \theta=\frac{1}{2} d^{\prime \prime} \Delta G \theta=\frac{1}{2} d^{\prime \prime} \theta=0$ so that $\partial^{\prime \prime} G d^{\prime \prime} \theta$ is a $d^{\prime \prime}$-closed $C^{\infty}$ form on $W^{\prime}$. The map $\theta \rightarrow \partial^{\prime \prime} G d^{\prime \prime} \theta$ is continuous because $G_{0}$ as defined in (2.5) maps bounded sets of test functions (with compact support) to bounded sets of test functions (with arbitrary support).

$\partial^{\prime \prime} G d^{\prime \prime} \theta$ is a $\bar{\partial}$-closed $C^{\infty}$ form. We must construct continuously a $C^{\infty}$ form $R$ such that $d^{\prime \prime} R=\partial^{\prime \prime} G d^{\prime \prime} \theta$, but this continuity must be in the distribution topology.

Adapt the proof of [5, Theorem I.D.3, p. 27].

To solve the equation $\partial g / \partial \bar{z}=f$ continuously for distributions (with $f$ and $g C^{\infty}$ functions), let $\eta$ be a function of $z$ alone which is identically 1 on $(1-\varepsilon) W^{\prime}$ and has compact support when restricted to the $z$-plane.

$$
g(z)=\frac{1}{2 \pi i} \iint \frac{\eta(\zeta) f(\zeta) d \zeta \wedge d \bar{\zeta}}{\zeta-z}
$$


satisfies $\partial g / \partial \bar{z}=f$ on $(1-2 \varepsilon) S$ and depends continuously on $f$. Using the contruction of [7, Theorem I.D.3, p. 27] with the above choice of $g$, we can construct $R$ such that $d^{\prime \prime} R=\partial^{\prime \prime} G d^{\prime \prime} \theta . R$ depends continuously on $\partial^{\prime \prime} G d^{\prime \prime} \theta$ and is defined on $W=(1-v \varepsilon) W^{\prime}$ for some $v$ depending only on the dimension $m$ of the simplex and the degree of $\partial^{\prime \prime} G d^{\prime \prime} \theta$.

On $W$, let $s=2\left(\partial^{\prime \prime} G \theta+R\right)$. From (2.6), on $W, t=d^{\prime \prime} s$. The map $t \rightarrow s$ is continuous as desired.

We can now return to the proof of Lemma 2.5. We are assuming that the simplex $D$ is equivalent to a domain in $C^{m}$. Let $\mathfrak{B}_{1}=\left\{V_{1, i}\right\}$ be a locally finite cover of $D$ by relatively compact polyrectangles such that the intersection of polyrectangles is a polyrectangle. Expand the $V_{1, i}$ slightly to form $V_{2, i}$ and the cover $\mathfrak{B}_{2}$. Expand the $V_{2, i}$ and continue until reaching $\mathfrak{B}_{q}$. We observe that for $C^{q}\left(N\left(\mathfrak{B}_{1}\right), \Omega^{p}\right), \delta_{q}$ is a homomorphism since we have Fréchet spaces. Now use diagram (2.2) for $D$, but for the left two columns use $\mathfrak{B}_{1}$, for the third $\mathfrak{B}_{2}$, for the fourth $\mathfrak{B}_{3}$ and so on. Let $\xi \in \Gamma\left(D, K^{p, q}\right)$ be a current such that $\bar{\partial} \xi=0$. Let

$$
\Lambda_{q-v-1}=\bar{\partial}_{v}^{-1} \circ \delta_{q-v-2} \circ \bar{\partial}_{v+1}^{-1} \circ \ldots \circ \bar{\partial}_{q-1}^{-1} \circ i
$$

and

$$
\Lambda_{q}=\varepsilon^{-1} \circ \delta_{q-1} \circ \Lambda_{q-1},
$$

but for $\bar{\partial}^{-1}$ use the above continuous construction, making $\Lambda_{v}$ a continuous map. Let $\xi_{v}=\Lambda_{v}(\xi)$. Since $\delta_{q-1}$ is a homomorphism, there is a continuous map $\xi_{q} \rightarrow C^{q-1}\left(N\left(\mathfrak{B}_{1}\right), \Omega^{p}\right) / \operatorname{ker} \delta_{q-1}$. Let $\zeta_{q-1}+\varepsilon \operatorname{ker} \delta_{q-1}$ be the image of $\xi_{q}$ in $C^{q-1}\left(N\left(\mathfrak{B}_{1}\right), E^{p, 0}\right) / \varepsilon \operatorname{ker} \delta_{q-1}$. Now we continue the diagram chase to work our way back to $\Gamma\left(D, K^{p, q-1}\right)$, but we remain with the cover $\mathfrak{B}_{1}$. The $\xi_{v}$ still depend continuously on $\xi$ after restriction to $N\left(\mathfrak{B}_{1}\right)$.

$\delta_{q-1}\left(\zeta_{q-1}+\varepsilon \operatorname{ker} \delta_{q-1}\right)=\delta_{q-1} \xi_{q-1}$ so $\left(\xi_{q-1}-\zeta_{q-1}\right)+\varepsilon \operatorname{ker} \delta_{q-1}$ is in the image of $\delta_{q-2}$ by exactness. $\bar{\partial}_{0} \circ \delta_{q-2}^{-1}$ induces a continuous map $\left(\xi_{q-1}-\zeta_{q-1}\right)+\varepsilon \operatorname{ker} \delta_{q-1}$ $\rightarrow \zeta_{q-2}+\bar{\partial}_{0} \operatorname{ker} \delta_{q-2} \in C^{q-2}\left(N\left(\mathfrak{B}_{1}\right), K^{p, 1}\right) / \bar{\partial}_{0} \operatorname{ker} \delta_{q-2}$.

$\delta_{q-2}\left(\zeta_{q-2}+\bar{\partial}_{0} \operatorname{ker} \delta_{q-2}\right)=\delta_{q-2} \xi_{q-2}$ so that, just as above, we have a continuous map induced by

$$
\begin{aligned}
\bar{\partial}_{1} \circ \delta_{q-3}^{-1}:\left(\xi_{q-2}-\zeta_{q-2}\right) & +\bar{\partial}_{0} \operatorname{ker} \delta_{q-2} \\
& \rightarrow \zeta_{q-3}+\bar{\partial}_{1} \operatorname{ker} \delta_{q-3} \in C^{q-3}\left(N\left(\mathfrak{B}_{1}\right), K^{p, 2}\right) / \bar{\partial}_{1} \operatorname{ker} \delta_{q-3} .
\end{aligned}
$$

Eventually, we get a continuous map to

$$
\zeta_{0}+\bar{\partial}_{q-2} \operatorname{ker} \delta_{0} \in C^{0}\left(N\left(\mathfrak{B}_{1}\right), K^{p, q-1}\right) / \ddot{\partial}_{q-2} \operatorname{ker} \delta_{0} .
$$

$\delta_{0}\left[\left(\xi_{0}-\zeta_{0}\right)+\bar{\partial}_{q-2} \operatorname{ker} \delta_{0}\right]=0$ so that $\left(\xi_{0}-\zeta_{0}\right)+\bar{\partial}_{q-2} \operatorname{ker} \delta_{0}=i\left(\omega+\bar{\partial}_{q-2} \Gamma\left(D, K^{p, q-2}\right)\right)$ for some $\omega \in \Gamma\left(D, K^{p, q-1}\right)$ such that $\bar{\partial} \omega=\xi . \omega+\bar{\partial}_{q-2} \Gamma\left(D, K^{p, q-2}\right)$ depends continuously on $\zeta$, as desired.

This completes the proof of Theorem 2.1. Strictly speaking, for currents we have only proved the isomorphism for Leray coverings whose simplices have one 
coordinate system. However, via $C^{\infty}$ forms, we know that all Leray coverings yield the same topology.

3. The duality theorem. The proof of this theorem is essentially a modification of the proof of the universal coefficient theorem for cohomology. We keep track of the topological conditions. The term $R^{q+1}\left(D, \Omega^{p}\right)$, which corresponds to the Ext term, is, unfortunately, not defined functorially in terms of the cohomology groups. Also, our splitting map is not natural.

We recall [7, Theorem 5.11, p. 41] that if $S$ is a linear topological space, $F$ is the closure of $\{0\}$ and $G$ is an arbitrary subspace of $S$ which is complementary to $F$, then the relative topology for $G$ is Hausdorff and $S$ is topologically isomorphic to $G \times F$, where $F$ has the indiscrete topology. Also, if $S$ is locally convex, the weak closure of $\{0\}$ coincides with its strong closure [7, Theorem 17.1, p. 154] so that the above decomposition may also be used for the weak topology. We shall sometimes call $F$ the indiscrete part of $S$.

THEOREM 3.1. Let $D$ be a $\sigma$-compact, $n$-dimensional complex manifold. Then

$$
H^{n-q}\left(D, \Omega^{n-p}\right) \approx\left[H_{*}^{q}\left(D, \Omega^{p}\right)\right]^{*} \oplus R_{*}^{q+1}\left(D, \Omega^{p}\right)
$$

and

$$
H_{*}^{n-q}\left(D, \Omega^{n-p}\right) \approx\left[H^{q}\left(D, \Omega^{p}\right)\right]^{*} \oplus R^{q+1}\left(D, \Omega^{p}\right) .
$$

$R_{*}^{q+1}\left(D, \Omega^{p}\right)=0$ if and only if $H_{*}^{q+1}\left(D, \Omega^{p}\right)$ has a Hausdorff topology. $R^{q+1}\left(D, \Omega^{p}\right)=0$ if and only if $H^{q+1}\left(D, \Omega^{p}\right)$ has a Hausdorff topology.

If $H^{n-q}\left(D, \Omega^{n-p}\right)$ and $H_{*}^{n-q}\left(D, \Omega^{n-p}\right)$ are given the weak topologies, then (3.1) and (3.2) are topological isomorphisms when $\left[H_{*}^{q}\left(D, \Omega^{p}\right)\right]^{*}$ and $\left[H^{q}\left(D, \Omega^{p}\right)\right]^{*}$ are given the weak* topologies and $R^{q+1}\left(D, \Omega^{p}\right)$ and $R_{*}^{q}{ }^{+1}\left(D, \Omega^{p}\right)$ are given the indiscrete topologies.

If $H^{n-q}\left(D, \Omega^{n-p}\right)$ and $H_{*}^{n-q}\left(D, \Omega^{n-p}\right)$ are given the strong topologies, then (3.1) and (3.2) are topological isomorphisms when $\left[H_{*}^{q}\left(D, \Omega^{p}\right)\right]^{*}$ and $\left[H^{q}\left(D, \Omega^{p}\right)\right]^{*}$ are given the topologies of uniform convergence on bounded sets of cocycles and $R^{q+1}\left(D, \Omega^{p}\right)$ and $R_{*}^{q+1}\left(D, \Omega^{p}\right)$ are given the indiscrete topologies.

Proof. We shall only write out the proof for (3.2) in full. The proof for (3.1) is virtually the same as the proof for (3.2). $\Gamma\left(D, K^{p, q}\right)$ is the dual of $\Gamma_{*}\left(D, E^{n-p, n-q}\right)$ and $\Gamma_{*}\left(D, K^{p, q}\right)$ is the dual of $\Gamma\left(D, E^{n-p, n-q}\right)$. Moreover, the strong topologies agree and the spaces are reflexive.

For the moment, we give everything the strong topology and work with $C^{\infty}$ forms.

$$
\bar{\partial}_{q}: \Gamma\left(D, E^{p, q}\right) \rightarrow \Gamma\left(D, E^{p, q+1}\right)
$$

may be factored as $\bar{\partial}_{q}=j_{q+1} \circ i_{q+1} \circ \tilde{\partial}_{q}$. Here $\tilde{\partial}_{q}: \Gamma\left(D, E^{p, q}\right) \rightarrow B^{q+1}\left(D, \Omega^{p}\right)$ is the projection map of $\Gamma\left(D, E^{p, q}\right)$ onto $B^{q+1}\left(D, \Omega^{p}\right)=\Gamma\left(D, E^{p, q}\right) / \operatorname{ker} \bar{\partial}$, which is given the quotient topology. Thus $\tilde{\partial}_{q}$ is a homomorphism. $B^{q+1}\left(D, \Omega^{p}\right)$ is algebraically isomorphic to $\beta^{q+1}\left(D, \Omega^{p}\right)$, the coboundaries. We give $\beta^{q+1}\left(D, \Omega^{p}\right)$ the topology it 
receives as a subset of $Z^{q+1}\left(D, \Omega^{p}\right)$, the $q+1$ cycles. $i_{q+1}: B^{q+1}\left(D, \Omega^{p}\right) \rightarrow Z^{q+1}\left(D, \Omega^{p}\right)$ is continuous but not necessarily a homomorphism. $j_{q+1}: Z^{q+1}\left(D, \Omega^{p}\right)$ $\rightarrow \Gamma\left(D, E^{p, q+1}\right)$ is the injection of the cocycles into the cochains and is a homomorphism.

We have the following algebraically exact sequences

$$
\begin{aligned}
& 0 \longrightarrow Z^{q}\left(D, \Omega^{p}\right) \stackrel{j_{q}}{\longrightarrow} \Gamma\left(D, E^{p, q}\right) \stackrel{\tilde{\partial}_{q}}{\longrightarrow} B^{q+1}\left(D, \Omega^{p}\right) \longrightarrow 0, \\
& 0 \longrightarrow B^{q}\left(D, \Omega^{p}\right) \stackrel{i_{q}}{\longrightarrow} Z^{q}\left(D, \Omega^{p}\right) \stackrel{\pi_{q}}{\longrightarrow} H^{q}\left(D, \Omega^{p}\right) \longrightarrow 0 .
\end{aligned}
$$

(3.3) is topologically exact. $\pi_{q}$ is a homomorphism.

We claim that diagram (3.5) is commutative and algebraically exact except for the middle row.

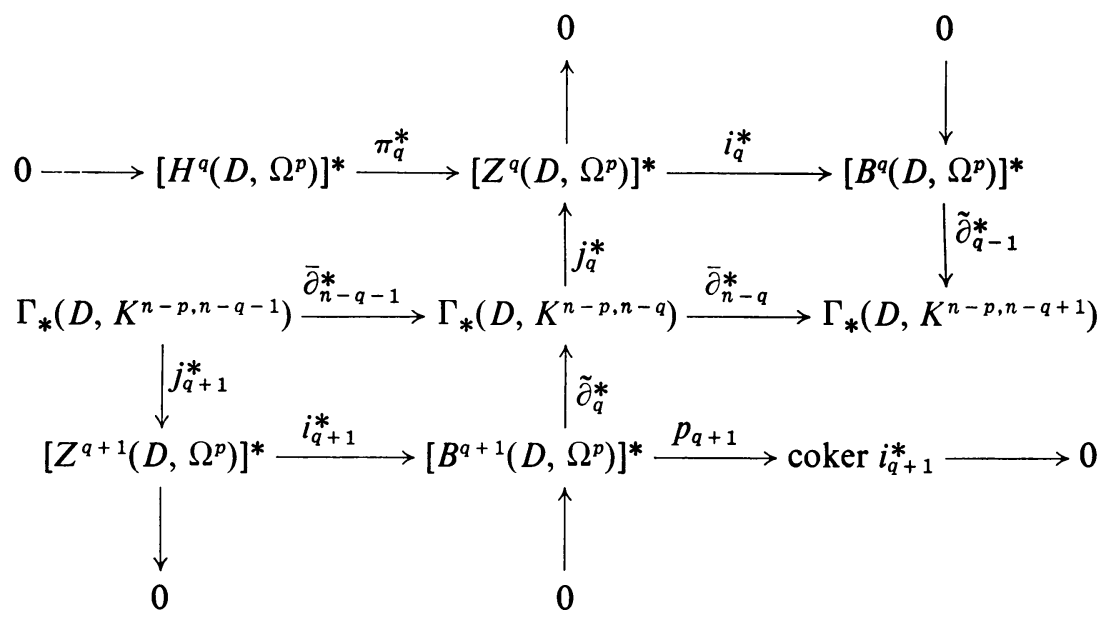

Commutativity follows from our factorization of $\bar{\partial}$ as $\bar{\partial}=j \circ i \circ \tilde{\partial}$. Exactness of the first row follows from the algebraic exactness of (3.4) and from the fact that $\pi_{q}$ is an open mapping. For the last row, exactness corresponds to the definition of cokernel as range/image [9, p. 12]. Exactness for the columns follows from the topological exactness of (3.3) and [7, Theorem 17.14, pp. 160-161]. Also, by [7, Theorem 17.14, pp. 160-161], $\pi^{*}, j^{*}$, and $\tilde{\partial}^{*}$ are all homomorphisms in the weak* topologies since they are adjoints of homomorphisms. Give coker $i_{q+1}^{*}$ the quotient topology, making $p_{q+1}$ a homomorphism.

We can chase diagram (3.5) as in $[9, \mathrm{p} .77]$ to obtain the following topologically exact sequence.

$$
0 \rightarrow \operatorname{coker} i_{q+1}^{*} \stackrel{\tilde{\partial}_{q}^{*} \circ p_{q+1}^{-1}}{\longrightarrow} H_{*}^{n-q}\left(D, \Omega^{n-p}\right) \stackrel{\left(\pi_{q}^{*}\right)^{-1} \circ j_{q}^{*}}{\longrightarrow}\left[H^{q}\left(D, \Omega^{p}\right)\right]^{*} \rightarrow 0 .
$$

$\tilde{\partial}_{q}^{*} \circ p_{q+1}^{-1}$ is continuous. Since im $\tilde{\partial}_{q}^{*}$ contains im $\bar{\partial}_{n-q-1}^{*}$,

$$
p_{q+1} \circ\left(\tilde{\partial}_{q}^{*}\right)^{-1}: \operatorname{im} \tilde{\partial}_{q}^{*} / \operatorname{im} \bar{\partial}^{*} \rightarrow \operatorname{coker} i_{q+1}^{*}
$$


is a continuous inverse for $\tilde{\partial}_{q}^{*} \circ p_{q+1}^{-1} .\left(\pi_{q}^{*}\right)^{-1} \circ j_{q}^{*}$ is continuous. $\left(j_{q}^{*}\right)^{-1} \circ \pi_{q}^{*}$ provides a continuous inverse.

To prove (3.2) let $R^{q+1}\left(D, \Omega^{p}\right)=$ coker $i_{q+1}^{*}$. For $(3.1)$, let $R_{*}^{q+1}\left(D, \Omega^{p}\right)$ $=$ coker $i_{*, q+1}^{*}$. Since we have vector spaces, (3.6) splits algebraically but we must still verify the topological conditions.

To show that coker $i_{q+1}^{*}$ receives the indiscrete topology, we must show that $i_{q+1}^{*}\left[Z^{q+1}\left(D, \Omega^{p}\right)\right]^{*}$ is dense in $\left[B^{q+1}\left(D, \Omega^{p}\right)\right]^{*}$. Let $f: B^{q+1}\left(D, \Omega^{p}\right) \rightarrow C$ be an element of $\left[B^{q+1}\left(D, \Omega^{p}\right)\right]^{*}$. We need only consider a weak * neighborhood $U$ of $f$ given by $U=\left\{g \in\left[B^{q+1}\left(D, \Omega^{p}\right)\right]^{*}|| f\left(x_{i}\right)-g\left(x_{i}\right) \mid<\varepsilon, 1 \leqq i \leqq s\right\}$. Define a function $\tilde{f}$ on the subspace $S$ spanned by the $x_{i}$ as $f\left(x_{i}\right)=f\left(x_{i}\right) . i_{q+1}(S) \subset Z^{q+1}\left(D, \Omega^{p}\right)$ is finite-dimensional and hence receives the usual topology as a subspace of $Z^{q+1}\left(D, \Omega^{p}\right)$ [7, Theorem 7.3, p. 59]. Then $\tilde{f}$ may be considered as a continuous linear functional on $i_{q+1}(S)$. Now extend $\tilde{f}$ to $F$, a continuous linear functional on all of $Z^{q+1}\left(D, \Omega^{p}\right) . i_{q+1}^{*} F \in U$, proving that coker $i_{q+1}^{*}$ does have the indiscrete topology.

Since $\left(\pi^{*}\right)^{-1} \circ j^{*}$ is continuous and $\left[H^{q}\left(D, \Omega^{p}\right)\right]^{*}$ has a Hausdorff topology, the image of coker $i_{q+1}^{*}$ is closed in $H^{n-q}\left(D, \Omega^{n-p}\right)$. Thus the image of coker $i_{q+1}^{*}$ is the closure of $\{0\}$. By [7, Theorem 5.11, p. 41], (3.2) is a topological decomposition. This proves (3.2) for the weak topology and as remarked above, a similar argument proves (3.1).

Before turning to the strong topologies, we note that the image of coker $i_{q+1}^{*}$ in $\Gamma_{*}\left(D, K^{n-p, n-q}\right)$ is necessarily the weak closure of $\bar{\partial}^{*}\left(\Gamma_{*}\left(D, K^{n-p, n-q-1}\right)\right)$ since coker $i_{q+1}^{*}$ maps onto the indiscrete part of $H_{*}^{n-q}\left(D, \Omega^{n-p}\right)$.

Now give all the duals except $\left[H^{q}\left(D, \Omega^{p}\right)\right]^{*}$ in diagram (3.5) the strong topology. $j_{q}^{*}$ remains an open mapping since $\Gamma\left(D, E^{p . q}\right)$ is reflexive [7, Theorem 20.2, p. 190]. $\tilde{\partial}_{q}^{*}$ is not assumed to be a homomorphism, although it is continuous. We give $\left[H^{q}\left(D, \Omega^{p}\right)\right]^{*}$ the topology it receives as a subset of $\left[Z^{q}\left(D, \Omega^{p}\right)\right]^{*}$ so that $\pi_{q}^{*}$ is a homomorphism. The topology on $\left[H^{q}\left(D, \Omega^{p}\right)\right]^{*}$ is then that of uniform convergence on bounded sets of cocycles.

(3.6) remains algebraically exact and $\left(\pi_{q}^{*}\right)^{-1} \circ j_{q}^{*}$ remains a homomorphism. coker $i_{q+1}^{*}$ has the same image in $\Gamma_{*}\left(D, K^{n-n, n-q}\right)$ as before, the weak closure of $\bar{\partial}^{*}\left(\Gamma_{*}\left(D, K^{n-p, n-q-1}\right)\right)$. But the weak closure of a subspace is the same as its strong closure since we are dealing with convex sets [7, Theorem 17.1, p. 154]. Thus, giving coker $i_{q+1}^{*}$ the indiscrete topology again makes $\tilde{\partial}_{q}^{*} \circ p_{q+1}^{-1}$ a homomorphism and it maps coker $i_{q+1}^{*}$ onto the strong closure of $\{0\}$ in $\left[H^{q}\left(D, \Omega^{p}\right)\right]^{*}$. Hence, we again have the direct sum decomposition of (3.1) and (3.2).

It remains to verify that $R^{q+1}\left(D, \Omega^{p}\right)=0$ if and only if $H^{q+1}\left(D, \Omega^{p}\right)$ has a Hausdorff topology, and that $R_{*}^{q+1}\left(D, \Omega^{p}\right)=0$ if and only if $H_{*}^{q+1}\left(D, \Omega^{p}\right)$ has a Hausdorff topology. Unlike as in the proof of (3.1) and (3.2), we must handle each case separately.

First consider $H^{q+1}\left(D, \Omega^{p}\right)$ and compute by $C^{\text {r }}$ forms with the strong topology. If $H^{q+1}\left(D, \Omega^{p}\right)$ has a Hausdorff topology, then $i_{q+1}\left(B^{q+1}\left(D, \Omega^{p}\right)\right)=\beta^{q+1}\left(D, \Omega^{p}\right)$ 
is necessarily closed in $Z^{q+1}\left(D, \Omega^{p}\right)$ and we have a continuous map of one Fréchet space onto another. Hence $i_{q+1}$ is a homomorphism and every function which is continuous on $B^{q+1}\left(D, \Omega^{p}\right)$ is continuous on $\beta^{q+1}\left(D, \Omega^{p}\right)$ and thus has a continuous extension to $Z^{q+1}\left(D, \Omega^{p}\right)$. Hence $i_{q+1}^{*}$ is onto and coker $i_{q+1}^{*}=0$.

Conversely, assume that $i_{q+1}^{*}$ is onto, which is equivalent to coker $i_{q+1}^{*}=0$. We want to show that $\beta^{q+1}\left(D, \Omega^{p}\right)$ is closed. We know that every continuous functional on $B^{q+1}\left(D, \Omega^{p}\right)$ is the image of some functional on $Z^{q+1}\left(D, \Omega^{p}\right)$ and since every continuous functional on $\beta^{q+1}\left(D, \Omega^{p}\right)$ extends to $Z^{q+1}\left(D, \Omega^{p}\right)$, the linear functionals on $B^{q+1}\left(D, \Omega^{p}\right)$ and on $\beta^{q+1}\left(D, \Omega^{p}\right)$ coincide. Thus $B^{q+1}\left(D, \Omega^{p}\right)$ and $\beta^{q+1}\left(D, \Omega^{p}\right)$ have the same weak topologies. Hence the injection $i_{q+1}: B^{q+1}\left(D, \Omega^{p}\right) \rightarrow Z^{q+1}\left(D, \Omega^{p}\right)$ is weakly relatively open. By [7, Theorem 21.9, p. 205], the image of $i_{q+1}$ is closed. Hence $H^{q+1}\left(D, \Omega^{p}\right)$ has a Hausdorff topology as asserted.

Now for $H_{*}^{q+1}\left(D, \Omega^{p}\right)$. Suppose that $H_{*}^{q+1}\left(D, \Omega^{p}\right)$ has a Hausdorff topology, then from (3.2), $R^{n-q}\left(D, \Omega^{n-p}\right)=0$. By our previous result, $H^{n-q}\left(D, \Omega^{n-p}\right)$ has a Hausdorff topology. By (3.1), we must have $R_{*}^{q+1}\left(D, \Omega^{p}\right)=0$. Now assume that $R_{*}^{q+1}\left(D, \Omega^{p}\right)=0$. By $(3.1), H^{n-q}\left(D, \Omega^{n-p}\right)$ has a Hausdorff topology. By our previous result, $R^{n-q}\left(D, \Omega^{n-p}\right)=0$. By (3.2), $H_{*}^{q+1}\left(D, \Omega^{p}\right)$ has a Hausdorff topology.

We see from Theorem 5.2 that Serre duality, $H_{*}^{n-q}\left(D, \Omega^{n-p}\right) \approx\left[H^{q}\left(D, \Omega^{p}\right)\right]^{*}$, occurs if and only if $R^{q+1}\left(D, \Omega^{p}\right)=0$, which occurs if and only if $H^{q+1}\left(D, \Omega^{p}\right)$ has a Hausdorff topology. But since we can compute $H^{q+1}\left(D, \Omega^{p}\right)$ by Fréchet spaces and we have an open mapping theorem for Fréchet spaces, $H^{q+1}\left(D, \Omega^{p}\right)$ has a Hausdorff topology if and only if $\bar{\partial}_{q}$ is a homomorphism.

Serre [15] works with analytic fiber spaces with vectorial fibers. These correspond to locally free sheaves. Thus we may choose a set of matrices $M^{\alpha \beta}$ which represent a locally free sheaf and proceed, following Serre, to define differential forms and currents. If $M^{\alpha \beta}$ represents the fiber space $V$, we let $V^{*}$ be the dual to $V$. The transformation matrices for $V^{*}$ are $\check{M}^{\alpha \beta}={ }^{t}\left(M^{\alpha \beta}\right)^{-1}$. Theorem 2.1 is true; its proof may be modified by working locally on those open sets where we have a trivial fiber space. The proof of Theorem (3.1) may be similarly modified to prove the following theorem.

THEOREM 3.2. Let $D$ be a $\sigma$-compact, $n$-dimensional complex manifold and let $V$ be an analytic fiber space with base $D$ with a vectorial fiber. Then

$$
H^{n-q}\left(D, \Omega^{n-p}\left(V^{*}\right)\right) \approx\left[H_{*}^{q}\left(D, \Omega^{p}(V)\right)\right]^{*} \oplus R_{*}^{q+1}\left(D, \Omega^{p}(V)\right)
$$

and

$$
H_{*}^{n-q}\left(D, \Omega^{n-p}\left(V^{*}\right)\right) \approx\left[H^{q}\left(D, \Omega^{p}(V)\right)\right]^{*} \oplus R^{q+1}\left(D, \Omega^{p}(V)\right) .
$$

$R_{*}^{q+1}\left(D, \Omega^{p}(V)\right)=0$ if and only if $H_{*}^{q+1}\left(D, \Omega^{p}(V)\right)$ has a Hausdorff topology. $R^{q+1}\left(D, \Omega^{p}(V)\right)=0$ if and only if $H^{q+1}\left(D, \Omega^{p}(V)\right)$ has a Hausdorff topology.

If $H^{n-q}\left(D, \Omega^{n-p}\left(V^{*}\right)\right)$ and $H_{*}^{n-q}\left(D, \Omega^{n-p}\left(V^{*}\right)\right)$ are given the weak topologies, then (3.7) and (3.8) are topological isomorphisms when $\left[H_{*}^{q}\left(D, \Omega^{p}(V)\right)\right]^{*}$ and 
$\left[H^{q}\left(D, \Omega^{p}(V)\right)\right]^{*}$ are given the weak * topologies and $R^{q+1}\left(D, \Omega^{p}(V)\right)$ and $R_{*}^{q+1}\left(D, \Omega^{p}(V)\right)$ are given the indiscrete topologies.

If $H^{n-q}\left(D, \Omega^{n-p}\left(V^{*}\right)\right)$ and $H_{*}^{n-q}\left(D, \Omega^{n-p}\left(V^{*}\right)\right)$ are given the strong topologies, then (3.7) and (3.8) are topological isomorphisms when $\left[H_{q}^{*}\left(D, \Omega^{p}(V)\right)\right]^{*}$ and $\left[H^{q}\left(D, \Omega^{p}(V)\right)\right]^{*}$ are given the topologies of uniform convergence on bounded sets of cocycles and $R^{q+1}\left(D, \Omega^{p}(V)\right)$ and $R_{*}^{q+1}\left(D, \Omega^{p}(V)\right)$ are given the indiscrete topologies.

For currents or $C^{\infty}$ forms, and with or without compact support, from its definition as coker $i_{q+1}^{*}$ and [7, Theorem 17.13, p. 160], $R^{q+1}\left(D, \Omega^{p}\right)=0$ if and only if $\bar{\partial}_{q+1}$ is a homomorphism in the weak topologies. Combining this with Theorem 2.1, we have the following result.

Proposition 3.3. Let $D$ be a $\sigma$-compact complex manifold. For currents and $C^{\infty}$ forms, with or without compact support, $\bar{\partial}$ is a homomorphism in the weak topologies if and only if it has closed range.

4. Applications to domains in Stein manifolds. If $D$ is a complex manifold, let $\mathcal{O}_{D}$ be the set of global holomorphic functions on $D$.

THEOREM 4.1. Let $D$ be a Riemann domain over a Stein manifold such that $\mathfrak{C}_{D}$ separates points. If $H_{*}^{q}\left(D, \Omega^{n}\right) 1 \leqq q \leqq n-1$ has a Hausdorff topology and is finitc dimensional and if $H_{*}^{n}\left(D, \Omega^{n}\right)$ has a Hausdorff topology, then $D$ is a Stein manifold.

Proof. $H^{q}(D, \mathcal{O}) \approx\left[H_{*}^{n-q}\left(D, \Omega^{n}\right)\right]^{*} 1 \leqq q \leqq n-1$ by Theorem 3.1. Thus $D$ is a Stein manifold by [8, Theorem 4.1$]$.

If $X=M-D$, where $D$ is an open set in a complex manifold $M$, we have the following exact sequence.

$$
\longrightarrow H_{*}^{q}\left(M, \Omega^{p}\right) \stackrel{j}{\longrightarrow} H_{*}^{q}\left(X, \Omega^{p}\right) \stackrel{\delta}{\longrightarrow} H_{*}^{q+1}\left(D, \Omega^{p}\right) \stackrel{i}{\longrightarrow} H_{*}^{q+1}\left(M, \Omega^{p}\right) \longrightarrow
$$

[4, Theorem 4.10.1, p. 190]. $i, j$, and $\delta$ may be given as follows. Given a $\bar{\partial}$-closed differential form (or current) $\xi \in \Gamma_{*}\left(D, E^{p, q}\right)$, let $i$ (cls $\xi$ ) be the cohomology class of $\xi$ where $\xi$ is extended by 0 to all of $M . j(\operatorname{cls} \zeta), \zeta \in H^{q}\left(M, \Omega^{p}\right)$ is defined by restricting $\zeta$ to $X$. Finally given cls $\omega \in H_{*}^{q}\left(X, \Omega^{p}\right)$, extend $\omega$ to any arbitrary $C^{\infty}$ form $\omega^{\prime}$ with compact support on $M$. $\bar{\partial} \omega^{\prime}$ vanishes on some neighborhood of $X$ and thus may be restricted to $D$ to represent a class in $H_{*}^{q+1}\left(D, \Omega^{p}\right)$.

Now assume that $M$ is a Stein manifold. $H_{*}^{q}\left(M, \Omega^{p}\right)=0$ for $0 \leqq q \leqq n-1$, so, algebraically

$$
H_{*}^{q}\left(X, \Omega^{p}\right) \approx H_{*}^{q+1}\left(D, \Omega^{p}\right), \quad 0 \leqq q \leqq n-2
$$

and the following sequence is algebraically exact.

$$
0 \longrightarrow H_{*}^{n-1}\left(X, \Omega^{p}\right) \stackrel{\delta}{\longrightarrow} H_{*}^{n}\left(D, \Omega^{p}\right) \stackrel{i}{\longrightarrow} H_{*}^{n}\left(M, \Omega^{p}\right) \stackrel{j}{\longrightarrow} H_{*}^{n}\left(X, \Omega^{p}\right) \longrightarrow 0 .
$$

We shall give $H_{*}^{q}\left(X, \Omega^{p}\right)$ the (nonintrinsic) topology that it receives from $H_{*}^{q-1}\left(D, \Omega^{p}\right)$ for $q \leqq n-2$, via (4.2), as a subspace of $H^{n}\left(D, \Omega^{p}\right)$ for $q=n-1$ and 
as a quotient space in (4.3) for $q=n$. (See Theorem 4.12.) We then have a weak and a strong topology. Since we shall only be concerned with Hausdorff properties, this distinction will not be necessary in general.

The following theorem includes one of Friedman's [3]. $p$ is held fixed.

Theorem 4.2. Let $D$ be a domain in a Stein manifold $M$ of dimension $\geqq 2$. Let $X=M-D$. Then

$$
\mathrm{I}_{*}\left(X, \Omega^{p}\right) \approx H^{1}\left(D, \Omega^{p}\right) .
$$

$H_{*}^{1}\left(D, \Omega^{p}\right)=0$ if and only if $X$ has no nonempty relatively open, compact subsets. $H_{*}^{1}\left(D, \Omega^{p}\right)$ always receives a Hausdorff topology. $X$ has no nonempty relatively open, compact subsets if and only if $H^{n-1}\left(D, \Omega^{p}\right)$ has the indiscrete topology.

Proof. $\Gamma_{*}\left(X, \Omega^{p}\right) \approx H_{*}^{1}\left(D, \Omega^{p}\right)$ by (4.2). We have the identity theorem for $\Omega^{p}$ since it is locally free. Consider a section $s \in \Gamma_{*}\left(X, \Omega^{p}\right)$. The support of $s$ is open since we have the identity theorem. The support of $s$ is also necessarily closed. Thus if $\Gamma_{*}\left(X, \Omega^{p}\right) \neq 0, X$ has a nonempty, relatively open compact subset. Conversely, suppose that $X$ has a nonempty relatively open compact (and hence closed) subset $X_{1} . X=X_{1} \cup X_{2}, X_{1} \cap X_{2}=\varnothing$. Let $U_{1}$ and $U_{2}$ be disjoint open neighborhoods of $X_{1}$ and $X_{2}$. Define $f \in \Gamma_{*}\left(X, \Omega^{p}\right)$ as zero on $X_{2}$ and as the restriction to $X_{1}$ of some nonzero element of $\Omega^{p}$ on $U_{1}$. (Since local coordinates are given by elements of $\mathcal{O}_{M}$, there are many global sections of $\Omega^{p}$.)

Consider the topology $T$ of pointwise convergence of all derivatives for the local coefficients in $\Gamma_{*}\left(X, \Omega^{p}\right) . T$ is a rather weak topology but it is Hausdorff. For consider a nonzero germ in $\Gamma_{*}\left(X, \Omega^{p}\right)$. By the identity theorem, at least one of its coefficients must have a nonzero power series expansion at some point of $X$. $X \neq \varnothing$, for otherwise $\Gamma_{*}\left(X, \Omega^{p}\right)=0$.

To show that $H_{*}^{1}\left(D, \Omega^{p}\right)$ has a Hausdorff topology, it suffices to show that $r: H_{*}^{1}\left(D, \Omega^{p}\right) \rightarrow \Gamma_{*}\left(X, \Omega^{p}\right)$ is continuous with the weak topology on $H_{*}^{1}\left(D, \Omega^{p}\right)$. The mapping $r$ may be given explicitly as follows. Choose a representative $\xi$ for the cohomology class. We may let $\xi$ be a $C^{\infty}$ form with compact support. Extend $\xi$ to be a $C^{\infty}$ form with compact support on $M$. Since $H_{*}^{1}\left(M, \Omega^{p}\right)=0$, there is a $C^{\infty}$ form $\omega \in \Gamma_{*}\left(M, \Omega^{p}\right)$ such that $\bar{\partial} \omega=\xi$. $\omega$ is in fact unique since if $\bar{\partial} \omega^{\prime}=\xi$, $\omega-\omega^{\prime}$ would be analytic with compact support and thus identically zero. Let $r(\operatorname{cls} \xi)=\operatorname{cls}\left(\left.\omega\right|_{X}\right)$.

Extending $\xi$ to all of $M$ is continuous in the weak topologies since a test current on $M$ restricts to a test current on $D$. $\omega$ depends continuously on $\xi$ by Proposition 3.3 .

Finally we must show that the restriction of $\omega$ to $X$ is continuous. $\omega$ is a $C^{\infty}$ differential form which is holomorphic in a neighborhood of $X$. Therefore, evaluation at a point of $X$ of some derivative of a coefficient of $\omega$ is just the restriction of a current to those $\omega$ holomorphic in a neighborhood of $X$. Thus the restriction map is continuous. $r$ is a continuous map since it is the composition of continuous maps. 
Since $H^{n-1}\left(D, \Omega^{p}\right) \approx\left[H_{*}^{1}\left(D, \Omega^{n-p}\right)\right]^{*} \oplus R_{*}^{2}\left(D, \Omega^{n-p}\right), \quad H^{n-1}\left(D, \Omega^{p}\right)$ has the indiscrete topology if and only if $H_{*}^{1}\left(D, \Omega^{n-p}\right) \approx 0$.

We have an isomorphism $H^{n-1}\left(D, \Omega^{n}\right) / R_{*}^{2}\left(D, \Omega^{n}\right) \approx[\Gamma(X, \mathcal{O})]^{*}$ so that any $\bar{\partial}_{-}$ closed $(n, n-1)$ form $\omega$ must represent an element of $\left[\Gamma_{*}(X, \mathcal{O})\right]^{*}$. This pairing can be given explicitly as follows.

Let $f \in \Gamma_{*}(X, \mathcal{O})$, so that $f$ is a holomorphic function defined on some open set $U \supset X$. Think of $f$ as a distribution. We assume for simplicity that $X$ is compact. Take $V$ to be the union of $C^{1}$ simplices and such that $X \subset V \subset \bar{V} \subset U$. To find the image of $f$ in $H_{*}^{1}(D, \mathcal{O})$ we must first extend $f$ to all of $M$. This can be done by forming the distribution $\tilde{f}$ which equals $f$ on $\bar{V}$ and vanishes outside of $\bar{V}$. $\bar{\partial} \tilde{f}$ has compact support in $D$.

$$
\langle\bar{\partial} \tilde{f}, \omega\rangle=\langle\tilde{f},-\bar{\partial} \omega\rangle=-\int_{\bar{V}} f \bar{\partial} \omega=-\int_{\bar{V}} \bar{\partial}(f \omega)=-\int_{\bar{V}} d(f \omega)=-\int_{\partial V} f \omega
$$

by Stokes' theorem.

Suppose that $X$ is a point $p$. Then $f \rightarrow f(p)$ is a continuous linear functional on $\Gamma(X, \mathcal{O})$ in the topology of pointwise convergence and hence a continuous linear functional on $H_{*}^{1}(X, \mathcal{O})$. Thus the functional $f \rightarrow f(p)$ must correspond to an element $\alpha^{\prime}$ of $H^{n-1}\left(M-p, \Omega^{n}\right)$. By [8, Lemma 3.1, p. 107], $H^{n-1}\left(M-p, \Omega^{n}\right)$ corresponds to Laurent series of the form

$$
\sum_{v_{1}, \ldots, v_{n}<0} a_{v_{1} \cdots v_{n}} z_{1}^{v_{1}} \cdots z_{n}^{v_{n}}
$$

which converge for $\left|z_{i}\right|>0$, all $i$. If the $z_{i}$ are local coordinates, $\left\langle z_{i} f, \omega\right\rangle=0$. Hence $\omega$ corresponds to $a / z_{1} \cdots z_{n}$ for some constant $a$. For $M=C^{n}$, by [8, (3.8)], $1 / z_{1} \cdots z_{n}$ corresponds, up to a multiplicative constant, to

$$
\frac{1}{r^{2 n}} \sum_{i=1}^{n} \bar{z}_{i} d \bar{z}_{1} \wedge d z_{1} \wedge \cdots \wedge \hat{d} \bar{z}_{i} \wedge d z_{i} \wedge \cdots \wedge d \bar{z}_{n} \wedge d z_{n}
$$

where $\hat{d} \bar{z}_{i}$ indicates that this term should be omitted from the wedge product. We have thus derived the Bochner-Martinelli integral formula [1, Theorem 6, p. 662] and $[10,(9)$, p. 280] up to a multiplicative constant. This constant may be evaluated by choosing a specific surface and using partitions of unity suitably adapted to the surface.

THEOREM 4.3 (BOCHNER-MARTINELLI). Let $z$ be a point in a region $V$ which is the union of $C^{1}$ simplices. Then for any function which is holomorphic on a neighborhood of $\bar{V}$,

$$
\begin{aligned}
f(z)= & \frac{(n-1) !}{(2 \pi i)^{n}} \int_{\partial V} \frac{f(\zeta)}{|\zeta-z|^{2 n}} \sum_{i=1}^{n}\left(\bar{\zeta}_{i}-\bar{z}_{i}\right) d \bar{z}_{1} \\
& \wedge d z_{1} \wedge \cdots \wedge \hat{d} \bar{z}_{i} \wedge d z_{i} \wedge \cdots \wedge d \bar{z}_{n} \wedge d z_{n} .
\end{aligned}
$$

The next theorem is weaker than a result of Malgrange [10, p. 236].

TheOREM 4.4. Let $D$ be a domain in a Stein manifold. Then $H^{n}\left(D, \Omega^{p}\right)=0$. 
Proof. By Theorem 3.1, $H^{n}\left(D, \Omega^{p}\right) \approx\left[H_{*}^{0}\left(D, \Omega^{n-p}\right)\right]^{*} \oplus R_{*}^{1}\left(D, \Omega^{n-p}\right)$. But $H_{*}^{0}\left(D, \Omega^{n-p}\right)=\Gamma_{*}\left(D, \Omega^{n-p}\right)=0$ since $D$ is not compact. Also $R_{*}^{1}\left(D, \Omega^{n-p}\right)=0$ since $H_{*}^{1}\left(D, \Omega^{n-p}\right)$ has a Hausdorff topology by Theorem 4.2.

THEOREM 4.5. Let $D$ be a domain in a Stein manifold. Then $H^{n-1}\left(D, \Omega^{p}\right)$ is either 0 or an infinite-dimensional vector space.

Proof. $H^{n-1}\left(D, \Omega^{p}\right) \approx\left[H_{*}^{1}\left(D, \Omega^{n-p}\right)\right]^{*} \oplus R_{*}^{2}\left(D, \Omega^{n-p}\right)$. If the image of a Fréchet space is of finite codimension in a Fréchet space, then the image is closed, [15, Lemma 2, p. 21]. Thus the indiscrete part of any $H^{q}\left(D, \Omega^{p}\right)$ is necessarily 0 or infinite-dimensional. Finally, by Theorem 4.2, $H_{*}^{1}\left(D, \Omega^{n-p}\right) \approx \Gamma_{*}\left(X, \Omega^{n-p}\right)$, which is either 0 or an infinite-dimensional Hausdorff space. Hence $\left[H_{*}^{1}\left(D, \Omega^{n-p}\right)\right]^{*}$ is either 0 or an infinite-dimensional space.

TheOREM 4.6. Let $D$ be a domain in a Stein manifold $M$ such that $X=M-D$ is compact. If $H^{q}\left(X, \Omega^{p}\right)=0$ for $q \geqq 1$, then $H^{q}\left(D, \Omega^{n-p}\right)=0$ for $1 \leqq q \leqq n-2$ and $H^{n-1}\left(D, \Omega^{n-p}\right)$ has a Hausdorff topology.

Proof. We may assume that $n \geqq 2$. From (4.2), $H_{*}^{q}\left(D, \Omega^{p}\right)=0$ for $2 \leqq q \leqq n-1$. Also $H_{*}^{1}\left(D, \Omega^{p}\right)$ has a Hausdorff topology by Theorem 4.2. From (4.3), we have that

$$
0 \longrightarrow H_{*}^{n}\left(D, \Omega^{p}\right) \stackrel{i}{\longrightarrow} H_{*}^{n}\left(M, \Omega^{p}\right) \longrightarrow 0
$$

is an exact sequence. $i$ is continuous since it is induced by extending currents with compact support on $D$ to all of $M$. Since $H_{*}^{n}\left(M, \Omega^{p}\right) \approx\left[H^{0}\left(M, \Omega^{p}\right)\right]^{*}$ has a Hausdorff topology, $H_{*}^{n}\left(D, \Omega^{p}\right)$ must have a Hausdorff topology. From Theorem 3.1,

$$
H^{q}\left(D, \Omega^{n-p}\right) \approx\left[H_{*}^{n-q}\left(D, \Omega^{p}\right)\right]^{*} \oplus R_{*}^{n-q+1}\left(D, \Omega^{p}\right) .
$$

$R_{*}^{n-q+1}\left(D, \Omega^{p}\right)=0$ for all $q$ since we have Hausdorff topologies on the $H_{*}^{n-q+1}\left(D, \Omega^{p}\right)$. Thus $H^{q}\left(D, \Omega^{n-p}\right)=0$ for $1 \leqq q \leqq n-2$ and $H^{n-1}\left(D, \Omega^{n-p}\right)$ has a Hausdorff topology.

THeOREM 4.7. Let $D$ be a domain in $M$, a Stein manifold. Let $X=M-D$. Then $M$ is the envelope of holomorphy of $D$ if and only if $H_{*}^{n-1}\left(X, \Omega^{n}\right)$ has the indiscrete topology and $H_{*}^{n}\left(X, \Omega^{n}\right)=0$.

Proof. First assume that $M$ is the envelope of holomorphy of $D$. Then $\mathcal{O}_{M} \approx \mathcal{O}_{D}$ and $\left[\mathcal{O}_{M}\right]^{*} \approx\left[\mathcal{O}_{D}\right]^{*}$. By Theorem 3.1, $H_{*}^{n}\left(D, \Omega^{n}\right) \approx\left[\mathcal{O}_{D}\right]^{*} \oplus R^{1}(D, \mathcal{O})$ and $H_{*}^{n}\left(M, \Omega^{n}\right)$ $\approx\left[\mathcal{O}_{M}\right]^{*}$. It thus suffices to show that $i$ restricted to $\left[\mathcal{O}_{D}\right]^{*}$ is bijective, for then the result follows from the exactness of (4.3).

The map $i:\left[\mathcal{O}_{D}\right]^{*} \rightarrow\left[\mathcal{O}_{M}\right]^{*}$ is given as follows (see Diagram 3.5 ) and is the adjoint of the restriction map ${ }^{O_{M}} \rightarrow 0_{D}$. Take a continuous linear functional $\phi$ on $\mathcal{O}_{D}$ and extend it to a continuous linear functional on the $C^{\infty}$ functions on $D$. This is a current $\xi$ with compact support. Now extend $\xi$ by 0 to be a current with compact support on $M . i$ is injective, for if $i(\phi)=0$, then $\xi$ is zero on $\mathcal{O}_{M}$ and thus 
zero on $\mathcal{O}_{D}$ since every element of $\mathcal{O}_{M}$ comes from $\mathcal{O}_{D}$. Thus $\phi=0$. $i$ is surjective, for given $g \in\left[\mathcal{O}_{M}\right]^{*}$ consider $g$ as an element of $\left[\mathcal{O}_{D}\right]^{*}$ and then apply $i$.

Conversely, if $H_{*}^{n}\left(X, \Omega^{n}\right)=0$ and $H_{*}^{n-1}\left(X, \Omega^{n}\right)$ has the indiscrete topology, (4.3) yields the following algebraically exact sequence.

$$
0 \longrightarrow H_{*}^{n-1}\left(X, \Omega^{n}\right) \stackrel{\delta}{\longrightarrow} H_{*}^{n}\left(D, \Omega^{n}\right) \stackrel{i}{\longrightarrow} H^{n}\left(M, \Omega^{n}\right) \longrightarrow 0 .
$$

Since $i$ is continuous, $\operatorname{ker} i$ contains the indiscrete part of $H_{*}^{n}\left(D, \Omega^{n}\right)$. Since $\delta H^{n-1}\left(X, \Omega^{n}\right)$ is the kernel and has the indiscrete topology, $\operatorname{ker} i=R^{1}\left(D, \Omega^{n}\right)$. Hence $i$ induces a bijection

$$
i:\left[H^{0}\left(D, \Omega^{0}\right)\right]^{*} \rightarrow\left[H^{0}\left(M, \Omega^{0}\right)\right]^{*} \text { or } i:\left[\mathcal{O}_{D}\right]^{*} \rightarrow\left[\mathcal{O}_{M}\right]^{*} .
$$

Now give $\left[\mathcal{C}_{D}\right]^{*}$ and $\left[\mathcal{C}_{M}\right]^{*}$ the strong topologies. $i$, as the adjoint of a continuous map, is continuous and thus has a closed graph. As duals of Montel Fréchet spaces, $\left[\mathcal{O}_{D}\right]^{*}$ and $\left[\mathcal{O}_{M}\right]^{*}$ are barrelled and fully complete, [7] and [13]. A closed graph theorem may be applied [13, Theorem 1, p. 10] to conclude that $i$ is a homomorphism. Instead of the closed graph theorem, we can apply an open mapping theorem [6, Corollary 11, p. 80] and conclude as before that $i$ is an open mapping. Now take duals again with the strong topologies. $i^{*}: \mathscr{O}_{. I} \approx \mathcal{O}_{D}$ since the spaces are reflexive.

Thus $M$ and $D$ have isomorphic Fréchet spaces of holomorphic functions. It now suffices to observe that $i^{*}$ agrees with the restriction map.

THEOREM 4.8. Let $X=M-D$ where $D$ is a domain in the connected Stein manifold $M$. Then $H_{*}^{n-1}\left(X, \Omega^{n}\right)$ has the indiscrete topology if and only if $\mathcal{O}_{M}$ is dense in $\mathcal{O}_{D}$. Also, $H_{*}^{n}\left(X, \Omega^{n}\right)=0$ if and only if the restriction map $\rho: \mathcal{O}_{M} \rightarrow \mathcal{O}_{D}$ is a homomorphism in the strong topologies.

Proof. The map $i: H_{*}^{n}\left(D, \Omega^{n}\right) \rightarrow H_{*}^{n}\left(M, \Omega^{n}\right)$ induces the map $i^{*}:\left[\mathcal{O}_{D}\right]^{*} \rightarrow\left[\mathcal{O}_{M}\right]^{*}$, and $i^{*}$ is the adjoint of $\rho$. If $\mathcal{O}_{M}$ is dense in $\mathcal{O}_{D}$ then $i^{*}$ is an injection since the values of a continuous linear functional on $\mathcal{O}_{D}$ are determined by its values on $\mathcal{O}_{M}$. If $\mathcal{O}_{M}$ is not dense in $\mathcal{O}_{D}$, let $T=\overline{\mathcal{O}}_{M} \varsubsetneqq \mathcal{O}_{D}$. Select a function $f$ which is 0 on $T$ and nonzero on $S$, a one-dimensional subspace of $\mathcal{O}_{D} . f$ is continuous on $T+S$. Extend $f$ to a continuous linear functional on $\mathcal{O}_{D} \cdot i^{*}(f)=0$, so that $i^{*}$ is not injective and $H^{n-1}\left(X, \Omega^{n}\right)$ cannot map only to the indiscrete part of $H_{*}^{n}\left(D, \Omega^{n}\right)$.

For the second part of the theorem, we first observe that $\rho$ is an injection by the identity theorem. If $\rho: \mathcal{O}_{M} \rightarrow \mathcal{O}_{D}$ is a homomorphism, then the adjoint map $i^{*}:\left[\mathcal{O}_{D}\right]^{*} \rightarrow\left[\mathcal{O}_{M}\right]^{*}$ is surjective by the Hahn-Banach theorem. If $i^{*}$ is surjective, by [7, Theorem $21.9(\mathrm{v})$, p. 206], $\rho$ is a homomorphism.

Theorem 4.9 (Runge). Let $D \subset E$ be domains in $C^{1}$. Then $\mathcal{O}_{E}$ is dense in $\mathcal{O}_{D}$ if and only if $E-D$ has no nonempty relatively open, compact subsets.

Proof. We apply Theorem 4.8. $X=E-D . H^{0}\left(X, \Omega^{n}\right) \approx \Gamma_{*}(X, \mathcal{O})$ which vanishes if and only if $X$ has no nonempty relatively open, compact subsets. 
In Theorem 4.7, we have been using (4.3) to give $H_{*}^{n-1}\left(X, \Omega^{n}\right)$ a topology. We can, however, give $H_{*}^{n-1}\left(X, \Omega^{n}\right)$ a natural topology, i.e., one which depends only on arbitrarily small neighborhoods of $X$, which has the same indiscrete part.

A $\bar{\partial}$-closed form or current $\omega$ represents a class in $H_{*}^{n}\left(D, \Omega^{n}\right)$ which is in the closure of zero if and only if $\omega$ vanishes when evaluated against all holomorphic functions on $D$. Suppose $\omega$ has support in the compact set $K$. We first wish to show that there is a subdomain $F$ which contains $K$, has compact closure and is such that $\omega$ vanishes on $\mathcal{O}_{F}$.

Suppose $D$ is a Riemann domain over $C^{n}$ such that $\mathcal{O}_{D}$ separates points. Suppose also that $D=\bigcup_{m=1}^{\infty} D_{m}$ with $D_{m} \subset D_{m+1}$. Let $E_{m}$ be the envelope of holomorphy of $D_{m}$. There are natural locally biholomorphic projection maps $\pi_{r s}: E_{s} \rightarrow E_{r}$ for $r \geqq s$ and $\pi: E_{s} \rightarrow C^{n}$, for all $s$. Let $E$ be the direct (inductive) limit of this system. $E$ has the structure of a Riemann domain over $C^{n}$ and we have projection maps $\pi_{s}: E_{s} \rightarrow E$.

Lemma 4.1. Let $C$ be a compact set in $E$. Then there is an $E_{q}$ and $C^{\prime} \subset E_{q}$ such that $\pi_{q}: C^{\prime} \rightarrow C$ is onto and is biholomorphic in a neighborhood of $C^{\prime}$.

Proof. Cover $C$ by a finite number of open polydiscs $\Delta_{r}$ such that for each $\Delta_{r}$ there is an $E_{r}$ and $\Delta_{r}^{\prime}$ with $\pi_{r}: \Delta_{r}^{\prime} \rightarrow \Delta_{r}$ biholomorphic. Suppose $p \in \Delta_{1} \cap \Delta_{2}$. Then $p_{1}^{\prime} \in \Delta_{1}$ and $p_{2}^{\prime} \in \Delta_{2}$ such that $\pi_{1}\left(p_{1}^{\prime}\right)=p$ and $\pi_{2}\left(p_{2}^{\prime}\right)=p$ are equivalent. Thus there is an $s$ and $p^{\prime} \in E_{s}$ such that $\pi_{s 1}\left(p_{1}^{\prime}\right)=\pi_{s 2}\left(p_{2}^{\prime}\right)=p^{\prime} . \pi_{s}\left(p^{\prime}\right)=p$. Since $\Delta_{1} \cap \Delta_{2}$ is connected, $\pi_{s 1}\left(\Delta_{1}^{\prime}\right) \cup \pi_{s 2}\left(\Delta_{2}^{\prime}\right)$ must be mapped biholomorphically onto $\Delta_{1} \cup \Delta_{2}$ by $\pi_{s}$. Next look at $\Delta_{1} \cup \Delta_{2} \cup \Delta_{3}$. $\Delta_{3} \cap\left(\Delta_{1} \cup \Delta_{2}\right)$ can have at most two components so that the above lifting process need only be performed twice in order to find an $E_{t}$ such that $\pi_{t 1}\left(\Delta_{1}^{\prime}\right) \cup \pi_{t 2}\left(\Delta_{2}^{\prime}\right) \cup \pi_{t 3}\left(\Delta_{3}^{\prime}\right)$ is mapped biholomorphically onto $\Delta_{1} \cup \Delta_{2} \cup \Delta_{3}$ by $\pi_{t}$. After a finite number of steps we can lift $\cup \Delta_{r}$, proving the lemma.

THEOREM 4.10. Let $D=\bigcup D_{m}, D_{m} \subset D_{m+1}$. Let $E_{m}$ be the envelope of holomorphy of $D_{m}$. Let $E$ be the direct limit of the $E_{m}$. Then $E$ is a Stein manifold and the envelope of holomorphy of $D$.

Proof. Let $d$ be the distance function on $E$ [5, p. 44]. By [5, Theorem IX.D.4, p. 283], $E$ is a Stein manifold if and only if $-\log d$ is plurisubharmonic. It suffices to prove that $-\log d$ is locally plurisubharmonic. Let $p=\pi_{s}\left(p_{s}\right)$. Then there is a neighborhood $U_{s}$ of $p_{s}$ which is mapped biholomorphically by $\pi_{s}$ onto a neighborhood $U$ of $p$. We claim that for $q_{s} \in U_{s}, d\left(\pi_{s}\left(q_{s}\right)\right)=\lim _{t \rightarrow \infty} d\left(\pi_{t s}\left(q_{s}\right)\right)$. Since we have a monotone sequence, this equality shows that $-\log d$ is plurisubharmonic. Given any compact polydisc $\Delta$ centered at $\pi_{s}\left(q_{s}\right)$, by Lemma 4.1 , there is a $t$ and polydisc $\Delta_{t} \subset E_{t}$ such that $\pi_{t}\left(\Delta_{t}\right)=\Delta$. Let $q_{t}^{\prime} \in \Delta_{t}$ be $\pi_{t}^{-1}\left(\pi_{s}\left(q_{s}\right)\right)$. Then $q_{t}^{\prime}$ and $q_{s}$ are equivalent. For some $w, \pi_{w s}\left(q_{s}\right)=\pi_{w t}\left(q_{t}^{\prime}\right)$. $\pi_{w t}$ maps $\Delta_{t}$ biholomorphically, so that $d\left(\pi_{s}\left(q_{s}\right)\right)$ $=\lim _{t \rightarrow \infty} d\left(\pi_{t s}\left(q_{s}\right)\right)$.

Thus $E$ is a Stein manifold.

Every holomorphic function on $D$ extends to a holomorphic function on $E_{m}$. 
$f\left(\pi_{s t}\left(p_{t}\right)\right)=f\left(p_{t}\right)$, so that $f$ is well defined on $E$. Hence $E$ is the envelope of holomorphy of $D$.

THEOREM 4.11. Let $D$ be a domain in a Stein manifold $M$ such that $D=\bigcup_{m=1}^{\infty} D_{m}$ with $D_{m} \subset D_{m+1}$. Then given any compact set $K \subset D$, there is an $m$ such that any $f \subset \mathcal{O}_{D_{m}}$ can be approximated by $\mathcal{O}_{D}$ uniformly on $K$.

Proof. First suppose that $M=C^{n}$. Let $E$ be the envelope of holomorphy of $D$ and $E_{m}$ the envelope of holomorphy of $D_{m}$. Since $E$ is a Stein manifold there exist nested subdomains $E_{v}$ such that $E=\bigcup E_{v}, E_{v}$ has compact closure, and $\mathcal{O}_{E}$ is dense in $\mathcal{O}_{E_{v}}$. Suppose $K \subset E_{v}$. Then any $f \in \mathcal{O}_{E_{v}}$ may be approximated by $\mathcal{O}_{E}$ uniformly on $K$. By Lemma 4.1, $\bar{E}_{v}$ is the biholomorphic image of some $\bar{E}_{v}^{\prime} \subset E_{m}$, for some $m$. Hence every holomorphic function $f$ on $D_{m}$ extends to a holomorphic function $f$ on $E_{m}$ and determines a function $f$ on $E_{v}$. This last $f$ may be approximated by $\mathcal{O}_{D}=\mathcal{O}_{E}$ uniformly on $K$.

For arbitrary $M$, embed $E$ as a submanifold of $C^{2 n+1}$. There exists a neighborhood $U$ of $E$ and a holomorphic retraction $\pi: U \rightarrow E$ [5, Theorem VIII.C.8, p. 257]. Let $D^{\prime}=\pi^{-1}(D)$ and $D_{m}^{\prime}=\pi^{-1}\left(D_{m}\right) . D^{\prime}=\bigcup D_{m}^{\prime}$ and $D_{m}^{\prime} \subset D_{m+1}$. Now given $K \subset D$, by our previous result there is an $m$ such that any $g \in \mathcal{O}_{D_{m}^{\prime}}$ can be approximated by $\mathcal{O}_{D^{\prime}}$ uniformly on $K$. Since $\pi$ is holomorphic, any $f \in \mathcal{O}_{D_{m}}$ induces $g=f \circ \pi \in \mathcal{O}_{D_{m}^{\prime}}$. $g$ equals $f$ on $D_{m}$ and can be approximated by $\mathcal{O}_{D^{\prime}}$. By restricting the approximating functions to $D$, we can approximate $f$ by $\mathcal{O}_{D}$.

Finally we can return to our discussion of the topology on $H_{*}^{n-1}\left(X, \Omega^{n}\right)$. We shall define two natural topologies, one using $C^{\infty}$ forms and one using currents. The former is derived from a strong topology, the latter from a weak one. Consider pairs $(U, K)$ such that $U$ is an open neighborhood of $X$ and $K$ is a closed subset of $U$ such that $X \cap K$ is compact. These form a directed system with $(U, K) \geqq\left(U^{\prime}, K^{\prime}\right)$ if $U \subset U^{\prime}$ and $U \cap K^{\prime} \subset K$. Let $\Gamma_{\#}\left(U, K ; E^{n, n-1}\right)$ be the $C^{\infty}$ forms defined on $U$ with support in $K$ and let $\Gamma_{\#}\left(U, K ; K^{n, n-1}\right)$ be the similarly defined currents. These yield cohomology groups $H_{\#}^{n-1}\left(U, K ; \Omega^{n}\right)$. There is a natural restriction map

$$
\rho(U, K),\left(U^{\prime}, K^{\prime}\right): H_{\#}^{n-1}\left(U^{\prime}, K^{\prime} ; \Omega^{n}\right) \rightarrow H_{\#}^{n-1}\left(U, K ; \Omega^{n}\right)
$$

and the inductive limit is algebraically isomorphic to $H_{*}^{n-1}\left(X, \Omega^{n}\right)$. Thus to put a natural topology on $H_{*}^{n-1}\left(X, \Omega^{n}\right)$ it suffices to put a natural topology on $\Gamma_{\#}\left(U, K ; E^{n, n-1}\right)$ or $\Gamma_{\#}\left(U, K ; K^{n, n-1}\right)$. Give the $C^{\infty}$ forms the topology of uniform convergence on compact sets of all derivatives. Give $\Gamma_{\#}\left(U, K ; K^{n, n-1}\right)$ the weak topology generated by all $C^{\infty}$ test forms $\omega$ on $U$ such that supp $\omega \cap K$ is compact. Call the topology derived from $C^{\infty}$ forms the strong inductive topology and the topology derived from currents the weak inductive topology.

THEOREM 4.12. The strong inductive topology for $H_{*}^{n-1}\left(X, \Omega^{n}\right)$ is stronger than the strong topology. The weak inductive topology is stronger than the weak topology. 0 has the same closure in all four topologies. For the inductive topologies, $\omega$ is in the 
closure of 0 in $H_{*}^{n-1}\left(X, \Omega^{n}\right)$ if and only if some representative of $\omega$ is in the closure of 0 for some $H_{\#}^{n-1}\left(U, K ; \Omega^{n}\right)$.

Proof. To show that the inductive topologies are stronger than the other topologies it suffices to show that the map $H_{*}^{n-1}\left(X, \Omega^{n}\right) \rightarrow H_{*}^{n}\left(D, \Omega^{n}\right)$ is continuous. We need only show that the map $Z_{\#}^{n-1}\left(U, K ; \Omega^{n}\right) \rightarrow H_{*}^{n}\left(D, \Omega^{n}\right)$ is continuous for all $(U, K)$. Construct a $C^{\infty}$ function $\rho$ with compact support on $U$ which is identically 1 in a neighborhood of $X \cap K$. Then given $\omega \in \Gamma_{\#}\left(U, K ; E^{n, n-1}\right)$ or $\omega \in \Gamma_{\#}\left(U, K ; K^{n, n-1}\right)$ such that $\bar{\partial} \omega=0, \bar{\partial}(\rho \omega)$ represents the image of $\omega$ in $H_{*}^{n}\left(D, \Omega^{n}\right)$ and depends continuously on $\omega$.

For the last parts of the theorem, let cls $\omega \in H_{*}^{n}\left(D, \Omega^{n}\right)$ be such that cls $\omega$ is in the indiscrete part. $\omega$ may be either a $C^{\infty}$ form or a current. Then $\omega$ vanishes on $\mathcal{O}_{D}$. Let $K$ be the support of $\omega$. By Theorem 4.11 , there is a relatively compact region $D_{m}$ such that $\mathcal{O}_{D}$ approximates $\mathcal{O}_{D_{m}}$ on $K$. Thus $\omega$ also vanishes on $\mathcal{O}_{D_{m}}$. Using the weak topologies, we have a net $\omega_{\alpha} \rightarrow \omega$, where $\omega_{\alpha}=\bar{\partial} \theta_{\alpha}$ and the $\theta_{\alpha}$ have compact support in $D_{m}$. Let $E^{\prime}$ be a relatively compact region in $M$ which is a Stein manifold and contains $D_{m}$. Then $\omega$ vanishes on $\mathcal{O}_{E^{\prime}}$ and hence is exact on $E^{\prime}$. $\omega=\bar{\partial} \theta . \bar{\partial} \theta_{\alpha} \rightarrow \bar{\partial} \theta$ on $E^{\prime}$. By Proposition $3.3, \theta_{\alpha}+\operatorname{ker} \bar{\partial} \rightarrow \theta+\operatorname{ker} \bar{\partial}$ in $\Gamma_{*}\left(E^{\prime}, K^{n, n-1}\right) /$ $\operatorname{ker} \bar{\partial}_{n-1}$ or in $\Gamma_{*}\left(E^{\prime}, E^{n, n-1}\right) / \operatorname{ker} \bar{\partial}_{n-1}$. Thus $\theta$ is in the weak closure of those $\theta_{\beta} \in \Gamma_{*}\left(E^{\prime}, K^{n, n-1}\right)$ or $\theta_{\beta} \in \Gamma_{*}\left(E^{\prime}, E^{n, n-1}\right)$ such that $\theta_{\beta}$ is the sum of a current (or form) with support in $D_{m}$ and a current (or form) which is $\bar{\partial}$-closed and has compact support in $E^{\prime}$. Thus $\theta$ must also be in the strong closure of the $\theta_{\beta}$. For $C^{\infty}$ forms we now use the strong topology. We retain the weak topology on the currents.

Take a compact set $H$ which is a neighborhood of $\bar{E}^{\prime}$. The map from $C^{\infty}$ forms with compact support in $E^{\prime}$ to $C^{\infty}$ forms with compact support in $H$ is continuous. Thus $\theta$ is the strong limit of a sequence $\theta_{v}$ of $C^{\infty}$ forms with compact support in $H$ such that each $\theta_{v}$ is the sum of a form with support in $D_{m}$ and an exact form on $H$. Restrict the $\theta_{v}$ and $\theta$ to $M-\bar{D}_{m}=U$, an open neighborhood of $X$. Each $\theta_{v}$ is exact on $U . H \cap U=H^{\prime}$ is the desired closed subset since $H \cap X$ is compact. Since $\theta_{v} \rightarrow \theta$ uniformly in all derivatives, $\theta_{v} \rightarrow \theta$ in $\Gamma_{\#}\left(U, H^{\prime} ; E^{n, n-1}\right)$ as desired. For currents we have, as above, that $\theta_{\alpha} \rightarrow \theta$ on $E^{\prime}$ and the $\theta_{\alpha}$ are exact on $U$. We need to show that $\theta_{\alpha} \rightarrow \theta$ in $\Gamma_{\#}\left(U, H^{\prime} ; K^{n, n-1}\right) .\left\langle\theta_{\alpha}, t\right\rangle \rightarrow\langle\theta, t\rangle$ for any $C^{\infty}$ test form on $E^{\prime}$. Let $s$ be a test form for $\Gamma_{\#}\left(U, H^{\prime} ; K^{n, n-1}\right)$. Since supp $s \cap H^{\prime}$ is compact, $s$ may be extended by 0 to all of $E^{\prime}$. Then $\left\langle\theta_{\alpha}, s\right\rangle \rightarrow\langle\theta, s\rangle$ since $s$ is now a test form on $E^{\prime}$.

\section{BIBLIOGRAPHY}

1. S. Bochner, Analytic and meromorphic continuation by means of Green's formula, Ann. of Math. 44 (1943), 652-673.

2. P. Dolbeault, Formes différentielles et cohomologie sur une variété analytique complexe. I, Ann. of Math. 64 (1956), 83-130.

3. A. Friedman, Cohomology with compact support for the analytic sheaf, (to appear).

4. R. Godement, Topologie algébrique et théorie des faisceaux, Hermann, Paris, 1958.

5. R. Gunning and H. Rossi, Analytic functions of several complex variables, Prentice-Hall, Englewood Cliffs, N. J., 1965. 
6. T. Husain, The open mapping and closed graph theorems in topological vector spaces, Oxford at the Clarendon Press, 1965.

7. J. Kelley, I. Namioka et al., Linear topological spaces, Van Nostrand, Princeton, N. J., 1963.

8. H. Laufer, On sheaf cohomology and envelopes of holomorphy, Ann. of Math. 84 (1966), 102-118.

9. S. MacLane, Homology, Springer-Verlag, Berlin, 1963.

10. B. Malgrange, Faisceaux sur des variétés analytiques-réelles, Bull. Soc. Math. France 85 (1957), 231-237.

11. E. Martinelli, Sulle estensioni della formula integrale di Cauchy alle funzioni analitiche di più variabile complesse, Ann. Mat. Pura Appl. (4) 34 (1953), 277-347.

12. G. de Rham, Variétés différentiables, Hermann, Paris, 1960.

13. A. Robertson and W. Robertson, On the closed graph theorem, Proc. Glasgow Math. Assoc. 3 (1956), 9-12.

14. L. Schwartz, Théorie des distributions, Hermann, Paris, 1957.

15. J.-P. Serre, Un théorème de dualité, Comment. Math. Helv. 29 (1955), 9-26.

Massachusetts Institute of Technology,

Cambridge, MassachusetTS 